\title{
Revealing the nature of electron correlation in transition metal complexes with symmetry-breaking and chemical intuition
}

\author{
James Shee, ${ }^{1, \text { a) }}$ Matthias Loipersberger, ${ }^{1}$ Diptarka Hait, ${ }^{1}$ Joonho Lee,${ }^{2}$ and Martin Head-Gordon ${ }^{1, b)}$ \\ ${ }^{1)}$ Kenneth S. Pitzer Center for Theoretical Chemistry, Department of Chemistry, University of California, Berkeley, \\ California 94720, USA \\ ${ }^{2)}$ Department of Chemistry, Columbia University, New York, New York 10027, \\ $U S A$
}

In this work we provide a nuanced view of electron correlation in the context of transition metal complexes, reconciling theoretical concepts such as spin and spatial symmetry breaking in single-reference methods with physically-transparent intuition from ligand-field and molecular orbital theories. These insights provide the tools to reliably diagnose multi-reference character, and our analysis reveals that while strong (i.e. static) correlation can be found in linear molecules (e.g. diatomics), weakly-bound, and antiferromagnetically coupled (monometal-noninnocent ligand, or multi-metal) complexes, it is rarely found in the ground-states of monotransition-metal complexes. This leads to a picture of static correlation that is no more complex for transition metals than it is, e.g., for organic biradicaloids. In contrast, the ability of organometallic species to form more complex interactions, involving both ligand-to-metal $\sigma$-donation and metal-to-ligand $\pi$-backdonation, places a larger burden on a theory's treatment of dynamic correlation. We hypothesize that chemical bonds in which inter-electron pair correlation is non-negligible cannot be adequately described by theories using MP2 correlation energies, and indeed find large errors vs experiment for carbonyl-dissociation energies from double-hybrid density functionals. A theory's description of dynamic correlation (and to a less important extent, delocalization error), which affects relative spin-state energetics and thus spin-symmetry breaking, is found to govern the efficacy of its use to diagnose static correlation.

\section{INTRODUCTION}

While it can be agreed that the ab initio modeling of transition metal chemistry is a long-sought goal, the field is marked by stark differences of assumptions and approach. There have been a number of promising benchmark studies on inorganic and organometallic complexes, and the widespread use of Density Functional Theory (DFT) in these chemical realms has led to many important discoveries. ${ }^{1,2}$ However, there is a growing list of publications which document alarmingly large errors for smaller transition metal systems with respect to validated experimental measurements, ${ }^{3-5}$ implying that smaller systems such as mono-metal diatomics are not necessarily simpler. Furthermore, despite the evident complexity of systems with multiple spin centers such as oxygen-bridged multi-metal clusters, ${ }^{6,7}$ some groups, perhaps due to the absence of another feasible methodological option and/or some encouraging benchmarks ${ }^{8}$, have chosen to use DFT to study multi-metal clusters such as the oxygen evolving complex in Photosystem II. ${ }^{9}$ Amid this backdrop, this work aims to provide an intuitive way of understanding the nature of electron correlation in various types of transition metal compounds, which can serve to inform the choice of a suitable quantum chemical method in computing properties of such systems.

There are a number of aspects relevant to transition metals that are either less or not at all relevant for typ-

\footnotetext{
a) Electronic mail: jshee@berkeley.edu

b) Electronic mail: mhg@cchem.berkeley.edu
}

ical organic molecules, such as relativistic effects, a balanced treatment of solvation for redox species, and the likely possibility of converging to extrema other than the global minimum depending on the choice of SCF algorithm and/or initial guess. Yet, as these are, in principle, well-defined problems with well-defined (albeit often not perfect) solutions, ${ }^{10-13}$ it is often the case that terms such as "strong correlation" and "multi-reference character" are used as generic explanations in the face of unsystematically erroneous (and thus, apparently mysterious) predictions.

So let us start with some accepted working definitions: By multi-reference (MR) character we mean that the wavefunction of a chemical system contains more than one determinant with significant weight. Strong correlation (SC) is not necessarily implied by MR character as these determinants may only interact very weakly (e.g. unrestricted (U) Hartree Fock ( $\mathrm{HF}$ ) solutions for $\mathrm{H}_{2}$ in the dissociation limit), but can be said to occur when the MR nature of a system leads to the breakdown or lack of convergence of single-reference (SR) perturbation theory (PT). Herein we focus only on MR character, also known as "static correlation." In the cases of stretched $\mathrm{H}_{2}$ and biradicaloids, static correlation can be encountered when HOMO-LUMO and singlet-triplet gaps approach (but do not exactly reach) zero. The simplest examples can be found in stretched $\mathrm{H}_{2}$ and biradicaloid molecules such as $\mathrm{O}_{2}$ or benzyne isomers, where the lowest energy singlet wavefunctions have substantial open-shell character and thus require two determinants for a proper qualitative description. Within DFT, Yang and co-workers have likened such a situation to a fractional spin error. ${ }^{14,15}$ Variational single-determinant methods will, in these 
cases, exhibit spin-symmetry breaking (SSB), with the expectation value of the spin operator, $\left\langle S^{2}\right\rangle$, between 0 and 1 (exactly 1 for dissociated $\mathrm{H}_{2}$ and a perfect biradical). A second aspect which leads to MR character arises from the need of a wavefunction to transform as an irreducible representation of the molecular point group. Variational optimization of a single-determinant wavefunction, here, can only yield one of at times multiple configurations which when superposed yield the correct symmetry, and spatial SB then occurs. ${ }^{16}$ Simple examples can be found in HF calculations of stretched diatomics such as $\mathrm{F}_{2}^{+} \cdot{ }^{17}$ While we note that independentparticle theories can also break other intrinsic symmetries of the electronic Hamiltonian, ${ }^{18,19}$ e.g. those related to complex conjugation and time-reversal, in this work we will focus on spin and, to a lesser extent, spatial symmetry breaking and their relationship to MR character.

Transition metal atoms with partially-filled valence d shells can exhibit substantial MR character due to the close energetic spacing of many low-lying electronic states, in contrast to the spectra of typical closed-shell organic compounds. ${ }^{20}$ Transition metal diatomics have been studied extensively in recent years, ${ }^{3,4,20-27}$ and recent precise experiments from Morse et. al. ${ }^{28}$ have enabled meaningful comparisons between theory and experiment. Errors in the computed bond dissociation energies vs experiment as large as $30 \mathrm{kcal} / \mathrm{mol}$ were reported from $\mathrm{DFT}$, and neither the "gold standard" $\operatorname{CCSD}(\mathrm{T})^{29}$ nor a MR variant ${ }^{30}$ showed reliable accuracy for all species. ${ }^{4}$ It appears that some diatomics were "easier" to treat than others; however, common MR diagnostics did not correlate with the accuracy of any method and were shown to point to inconsistent conclusions depending on the particular diagnostic, and furthermore typically lack physically interpretive value. ${ }^{4,31}$ Many of these systems were later investigated with quantum Monte Carlo methods (QMC), ${ }^{5}$ which yielded very accurate results vs experiment when consistently employing multi-determinant trial wavefunctions. This level of accuracy, but with respect to near-exact benchmark calculations, could only be attained via high orders of CC theory which are not scalable to larger systems. ${ }^{26}$ Ref. 26 implies, as might be expected, that higher orders of CC theory are needed to describe increasing numbers of bonds; however, there were many exceptions to this trend. Considering even the simplest bonding motif (i.e. a single bond) as found in metal-hydride diatomics, many glaring irregularities could be found (convergence issues or $\sim 23 \mathrm{~kJ} / \mathrm{mol}$ inaccuracies at the $\operatorname{CCSD}(\mathrm{T})$ level), and no explanation on simple physical grounds has been provided.

Metal complexes with higher coordination number are of relatively greater interest to chemists, as the coordination environment resembles that of realistic transition metal catalysts or active sites in biology. 3d-containing complexes with partially-filled d shells are of particular interest due to their earth-abundance, ${ }^{32}$ and, from the perspectives of electronic structure and reactivity, due to the competing accessibility of low spin (LS) and high spin
(HS) configurations. As they are non-linear, Jahn-Teller (JT) distortions ${ }^{33}$ occur to avoid unequal occupancy of degenerate orbitals, i.e. to break orbital degeneracies due to spatial symmetry. However, remaining MR character has been suggested in Ref. 34, as a way of rationalizing large errors vs experiment of SR methods. Indeed, within a set of apparently simple and similar metal cations from Ref. 35, B3LYP, B97, and DLPNO-CCSD(T) performed very well for a subset but very poorly for another. The elucidation of underlying reasons for such aberrant behavior, most notably for complexes such as $\mathrm{Fe}\left(\mathrm{NH}_{3}\right)_{4}^{+}$ and $\mathrm{Mn}\left(\mathrm{NH}_{3}\right)_{4}^{+}$, is a primary motivation for the present study. We then proceed to highlight MR character in states which are LS due to antiferromagnetic coupling, as found in mono-metal complexes with redox-noninnocent ligands and oxygen-bridged $\mathrm{Mn}(\mathrm{III}) / \mathrm{Mn}(\mathrm{IV})$ dimers.

To provide more detail on the suitability of commonlyused MR diagnostics for transition metal systems, Wilson and co-workers have explored metrics derived from $\mathrm{CC}$ and Configuration Interaction (CI), e.g. T1 and D1 (Frobenius and matrix 2-norm of singles amplitude $\mathrm{t}_{1}$ in restricted CCSD, respectively), $\mathrm{C}_{0}^{2}$ (square of the HF configuration in the CISD wavefunction, or of the leading configuration state function in active-space methods), and \% TAE (triples contribution to the atomization energy). Those authors conclude that $\mathrm{CC}$ diagnostics are insufficient, that $\mathrm{C}_{0}^{2}$ from the Complete Active Space Self-Consistent Field $\left(\mathrm{CASSCF}^{36}\right)$ method is only reliable when a full active space can be considered, and that \%TAE fails for weakly-bound systems like $\mathrm{Zn}_{2}$. No linear correlation between any investigated diagnostics and the accuracy of the ccCA-TM composite method $^{37}$ was found. ${ }^{31}$ We note that even if they were reliable, diagnostics based on CCSD amplitudes and the relative contribution of the $(\mathrm{T})$ component would require calculations that scale as the 6th and 7th power of system size, respectively.

The applicability of CASSCF (e.g. in Ref. 38) is limited because exact CI solvers are feasible only for systems with less than $\sim 24$ active orbitals. ${ }^{39}$ Approximate solvers, e.g. $\mathrm{DMRG}^{40}$ or selected $\mathrm{CI}^{41-44}$, enable the use of a larger number of orbitals ${ }^{45,46}$, yet they are still sensitive to active space specification and nonetheless still scale exponentially. This active-space dependence renders CASSCF calculations, including RASSCF methods, ${ }^{47}$ rather difficult to properly converge. More generally, misleading conclusions can be drawn when active spaces are not sufficiently large. ${ }^{40}$ Ref.s 48 and 49 illustrate this difficulty with regard to the $\mathrm{MoFe}_{7} \mathrm{~S}_{9} \mathrm{C}$ catalytic center of nitrogenase. Furthermore, the delicate interplay between MR character and dynamic correlation can sometimes make the choice of sufficiently large active spaces quite unintuitive (e.g. active spaces with double d shells).

For the ground-state (GS) of non-linear molecules (with geometries optimized without symmetry constraints), we will explore in this work the use of SSB in unrestricted single-determinant wavefunctions formed 
from orbitals from Kohn-Sham DFT or $\kappa$-regularized orbital-optimized MP2 $(\kappa \text {-UOOMP2 })^{50}$ to detect the presence of MR-character in transition metal complexes. It is known ${ }^{51}$ that DFT orbitals, which are optimized in the presence of mean-field electron correlations, do not break spin or spatial symmetries as easily as HF orbitals. With regard to $\kappa$-UOOMP2, the inclusion of pair-wise additive electron correlation in the orbital optimization has a similar effect, and has been used to diagnose MR character in organic molecules and fullerenes ${ }^{52,53}$ and in one transition metal containing system (neutral iron porphyrin $)^{54}$. Moreover, given the plethora of MR diagnostics, we take a more appealing route using singleparticle orbital theory, drawing on chemical concepts from ligand-field and molecular orbital theories. ${ }^{55-57} \mathrm{We}$ note that these models have recently been connected with sophisticated $a b$ initio quantum-chemical methods, and successfully applied to a variety of experimental observations. ${ }^{58,59}$ We take one step further now, and seek to explore how ligand-field theoretical arguments can be used to understand and predict MR character, in conjunction with the quantitative use of SSB in SR theories that include some electron correlations. Thus, one main goal of this work is to provide, through a series of illustrative and chemically relevant examples, a link between chemical intuition and the occurrence of a MR wavefunction.

Correlation is often partitioned into "static" and "dynamic" contributions. If we assume, as most do, that the former is synonymous with MR character, there remains the question of which type of correlation is responsible for the bulk of the errors of commonly used SR quantum chemical methods. Admittedly, the extent to which static correlation is relevant for transition metal complexes is, at present, unclear. It is often assumed that there is a large degree of dynamic correlation, but can this quantity be connected with physical properties of the bonding exhibited by transition metal compounds? If so, what order of Moller Plesset (MP) PT or Coupled Cluster (CC) theory is needed? We endeavor to propose a more nuanced description of electron correlation than the strong vs weak distinction which at present permeates the field, which we hope will guide practitioners in their selection of appropriate quantum-chemical methods. As an example we focus on decidedly SR metal-carbonyl complexes, and suggest that the pair-wise additive correlation energy expression of MP2 is inadequate for the quantitative description of dative interactions consisting of $\sigma$ donation and $\pi$-backbonding, and thus caution against the use of MP2-based double-hybrid density functionals (DHDFs) for organometallic complexes.

\section{THEORETICAL OVERVIEW}

\section{A. Methods}

A variational theory is one which minimizes

$$
E=\frac{\langle\Psi|\hat{H}| \Psi\rangle}{\langle\Psi \mid \Psi\rangle}
$$

with respect to one or more parameters. In HF theory, $\Psi$ is a Slater determinant (antisymmetrized occupied orbitals) and the energy is minimized with respect to the orbital coefficients. In the CASSCF method, $\Psi$ is a linear combination of Slater determinants constructed from all possible electron configurations within a specified set of orbitals, called the active space. Both the CI and orbital coefficients are treated as variational parameters. A CASSCF calculation with the full orbital space as the active space is equivalent to full CI (FCI), and is exact within the basis set employed. Approximate FCI calculations are performed with the Adaptive Sampling Configuration Interaction (ASCI) method, ${ }^{43,60}$ a selected CI approach that iteratively improves a fixed-size CI wavefunction by selecting the most important configurations in the Hilbert space (via a first order PT based selection rule). Convergence of observables like energy or molecular properties can be gauged by comparing results from ASCI wavefunctions of increasing size, or by considering the second order perturbative correction ${ }^{61}$ to the ASCI energy.

$\kappa$-OOMP2 introduces a regularizer to OOMP2 (which optimizes orbitals to minimize $E_{0}+E^{(2)}$, where $E^{(2)}$ is not variational) to mitigate problems associated with vanishing HOMO-LUMO gaps in MP2, and is presented and discussed in detail in Ref.s 50 and 52. The $\kappa$-MP2 total energy, which is minimized with respect to orbital rotations in $\kappa$-OOMP2, is:

$$
E_{\kappa-M P 2}(\kappa)=E_{0}-\frac{1}{4} \sum_{i j a b} \frac{|\langle i j|| a b\rangle|^{2}}{\Delta_{i j}^{a b}}\left(1-e^{-\kappa \Delta_{i j}^{a b}}\right)^{2}
$$

where $i, j$ and $a, b$ represent occupied and virtual orbitals, respectively. $\Delta_{i j}^{a b}=\epsilon_{a}+\epsilon_{b}-\epsilon_{i}-\epsilon_{j}$, and the antisymmetrized two-electron integrals $\langle i j \| a b\rangle$ are defined as $\langle i j \mid a b\rangle-\langle i j \mid b a\rangle$. Eq. 2 reveals that $E_{\kappa-M P 2}(\kappa \rightarrow$ $0)=E_{H F}$. SSB in the HF solution can occur even for stable molecules at equilibrium bond lengths that should be well-described by a closed-shell configuration. This situation has been termed "artificial" SSB. ${ }^{52}$ In the limit of $\kappa \rightarrow \infty$, unregularized oo-MP2 is obtained, which does not have Coulson-Fischer (CF) points due to divergences accompanying small HOMO-LUMO gaps. ${ }^{62}$ Therefore, in this limit the theory is strongly biased in favor of spin symmetry restoration. As the tendency to break spin symmetry clearly depends on the choice of regularization parameter, an optimal $\kappa$ has been chosen in light of two criteria. The first is physically motivated, requiring $\mathrm{CF}$ points for single, double, and triple carbon-carbon 
bonds to occur at increasing bond distances. The second is empirically motivated, selecting $\kappa$ such that errors for reference reaction data sets are minimized: for thermochemistry, this leads to $\kappa=1.45 E_{h}^{-1} .50$

We note that a situation analogous to the latter is relevant to the specification of global hybrid DFT functionals, with the exchange part of the correlation energy given as:

$$
E_{x}=a E_{x}^{H F}+(1-a) E_{x}^{S L}
$$

where $E_{x}^{H F}$ is exact HF exchange (EXX) and $E_{x}^{S L}$ is the semilocal exchange functional. The SSB behavior of a global hybrid can be modulated by the fraction of EXX employed, i.e. the parameter $a$. One advantage of $\kappa$-UOOMP2 vs DFT is that the former is free of self-interaction error, in which an electron can spuriously interact with itself, and its many-body generalization known as delocalization error (DE) ${ }^{63,64}$ It is wellknown that DE tends to reduce the extent of SSB (and spatial SB) with pure and global hybrid (i.e. those with a modest amount of EXX, e.g. B3LYP) functionals in cases such as stretched HF, and is also relevant to the description of ligand-to-metal dative bonding.

\section{B. Calculation of $S^{2}$}

In 1955, Lowdin derived ${ }^{65}$ that

$$
\left\langle S^{2}\right\rangle=-\frac{N(N-4)}{4}+\int \Gamma\left(\mathbf{r}_{1} s_{1}, \mathbf{r}_{2} s_{2} \mid \mathbf{r}_{1} s_{2}, \mathbf{r}_{2} s_{1}\right) d \mathbf{x}_{1} d \mathbf{x}_{2}
$$

where the two-particle density matrix, $\Gamma$, is normalized to $\frac{N(N-1)}{2}$. Expressions of the two-particle density matrix are known for ROHF- and UHF-based theories along with simple density functional theories ${ }^{66,67}$. The UHF expression is of particular relevance to the present work, and can be written

$$
\left\langle S^{2}\right\rangle_{U H F}=S(S+1)+N_{\beta}-\sum_{i \bar{j}}^{o c c} S_{i \bar{j}}^{2}
$$

where $S=\frac{N_{\alpha}-N_{\beta}}{2}$ and $S_{i \bar{j}}$ is the $\alpha \beta$ overlap integral between the ith spin-up orbital and the jth spin-down orbital. We emphasize that the $\left\langle S^{2}\right\rangle$ value reported for DFT calculations is exact only for the fictitious noninteracting Kohn-Sham system, since the occupied KS orbitals are used instead of those from UHF to compute Eq. 5.

For $\kappa$-UOOMP2 and UOOCCD, unless otherwise mentioned, Eq. 5 is used with the orbitals resulting from the minimization procedure. A rigorous expression for $\left\langle S^{2}\right\rangle_{U O O C C D}$ can be found in Ref. 68. Regarding $\kappa$ UOOMP2, with the first-order MP wavefunction $\psi_{1}$ in hand, the expectation value of the spin operator can be rigorously obtained as

$$
\left\langle S^{2}\right\rangle_{M P 2}=\left\langle\psi_{0}\left|S^{2}\right| \psi_{0}\right\rangle+2\left\langle\psi_{0}\left|S^{2}\right| \psi_{1}\right\rangle
$$

assuming real amplitudes and orbitals. Natural orbital occupation numbers (NOONs) can be obtained by diagonalizing the 1-particle density matrix formed from $\psi_{1}$. As can be seen from Eq. 2, the amplitudes of the doublyexcited states in the $\kappa$-OOMP2 wavefunction are, by construction, encouraged to be small (i.e., when $\Delta_{i j}^{a b}$ is small, so is the regularizing term in parenthesis and thus the regularized amplitude). Thus, we expect that in most cases, $\left\langle S^{2}\right\rangle$ will be similar when computed either by Eq. 5 from $\kappa$-UOOMP2 orbitals, or by Eq. 6 with the perturbed wavefunction. Similar considerations apply to the NOONs.

\section{Computational Details}

All calculations were performed with Q-Chem 5.2, ${ }^{69}$ except for CASSCF calculations and geometry optimizations for $\mathrm{Fe}(\mathrm{II}) \mathrm{X}_{6}$ and the metal carbonyls not in Ref. 34 , which were performed with Orca. ${ }^{70}$ These geometry optimizations utilized the DKH Hamiltonian and -DK basis sets, for consistency with previous work. ${ }^{34}$ CASSCF calculations were initialized from DFT orbitals (in most cases, obtained with the B3LYP functional). For the ASCI calculations, we use approximate $\mathrm{NOs}^{43,60}$ or MCSCF-like orbital optimization within the active space $^{71}$, which leads to more compact CI wavefunctions and thus lower variational energies for a given wavefunction size. Further details about ASCI can be found in Refs 43,60 , and 61 . ASCI NOONs were computed from the variational CI wavefunction alone, without any perturbative corrections. For ASCI calculations of the HF molecule and transition metal hydrides, the second-order $\mathrm{PT}$ correction to the energy $\left(E_{P T 2}\right)$ is smaller than $10^{-3}$ Ha (i.e. $0.6 \mathrm{kcal} / \mathrm{mol}$ ) in magnitude. This high level of convergence was not possible for para-benzyne, and so we used the full-valence active space of 28 orbitals. The NOONs are converged by 4 million ASCI determinants (as shown in the Supporting Information).

To obtain $\left\langle S^{2}\right\rangle$ values and NOONs, the def2-SV(P) basis set is used ${ }^{72}$ (unless mentioned otherwise). UOOCCD calculations use the frozen-core approximation. While we are well-aware that for ground-state properties quantitative accuracy of energetic quantities vs experimental measurements requires much larger basis sets, the def2$\mathrm{SV}(\mathrm{P})$ basis is found to produce the qualitative descriptions required, and at a much-reduced computational cost that would be expected of a diagnostic tool.

The GDM algorithm ${ }^{73}$ is used as the default SCF solver for HF, DFT, $\kappa$-UOOMP2, and UOOCCD calculations. We confirmed the stability, with respect to orbital rotations, of HF and DFT solutions. For the $\kappa$-UOOMP2 calculations, the resolution of the identity approximation was employed with the corresponding auxiliary basis set, and, as discussed below, at times the DIIS solver was required. The integration grid for DFT calculations consists of 99 radial spheres each with 590 points. ${ }^{74}$

We employed an energy decomposition analysis (EDA) 
based on absolutely localized molecular orbitals to decompose the binding energies. The original scheme decomposes the interaction energy into frozen, polarization, and charge transfer contributions. ${ }^{75,76}$ In order to reveal more insight into the nature of the bidirectional charge transfer of some metal-ligand bonds, we augmented the analysis with a further decomposition of the CT energy using the newly developed variational forward-backward (VFB) decomposition ${ }^{77}$.

\section{RESULTS}

\section{A. Stretched $\mathbf{H}_{2}$, para-benzyne, and stretched HF}

In this section, we start from the simplest system that exhibits static correlation, stretched $\mathrm{H}_{2}$. Analytical expressions for $\mathrm{H}_{2}{ }^{78}$ show that the lowest unoccupied natural orbital (LUNO) occupation number for a single Slater determinant is related to $\left\langle S^{2}\right\rangle$ by:

$$
n_{L U N O}=1-\sqrt{1-\left\langle S^{2}\right\rangle} .
$$

In Fig. 1 we plot $n_{L U N O}$ vs bond length for three DFT functionals with varying amounts of $\operatorname{EXX}(0,20$, and $50 \%$ for BLYP, B3LYP, B5050LYP, respectively), HF, and $\kappa$ UOOMP2 vs exact results (obtained via CISD). The exact results shows fractional occupation of the antibonding orbital even at short bond lengths, whereas the other methods have zero occupation of the LUNO until beyond the Coulson-Fischer (CF) point, where spin-symmetry breaks. ${ }^{79}$ The $\mathrm{CF}$ point is pushed to longer bond lengths going from HF, $\kappa$-UOOMP2, to more pure DFT functionals. While all methods provide a satisfactory description of $\mathrm{H}_{2}$ in the dissociation limit, we note that in the region beyond $\sim 1.5 \AA, n_{L U N O}$ as approximated using DFT orbitals appears to be closer to the exact value than when (rigorously) derived from HF and $\kappa$-UOOMP2 theories.

As a second test, we consider para-benzyne, a prototypical biradicaloid. ${ }^{80,81}$ Using the cc-pVDZ basis and the geometry from Ref. 82, we compute reference HONO and LUNO populations with full valence CASSCF (28 electrons in 28 orbitals, using the ASCI solver ${ }^{71}$ ), which are compared with values from other methods in Fig. 2. As found above for $\mathrm{H}_{2}$, adding exact $\mathrm{HF}$ exchange systematically shifts the DFT predictions toward the HF result. We note that both B3LYP and $\kappa$-UOOMP2 produce NOONs in good agreement with the ASCI estimates.

While the above two examples would suggest that B3LYP is an excellent choice to describe SSB and fractional NOONs in these MR situations, its susceptibility to DE warrants caution in polar systems such as stretched hydrogen fluoride. In HF theory, the oneparticle self-interaction error is cancelled exactly - this is also the case in MP2 theories. Fig. 3 shows that the LUNO occupation at the B3LYP level at long bond lengths is significantly lower than the ASCI value, due to DE leading to contamination of the unrestricted solution with closed-shell, ionic contributions. Adding a

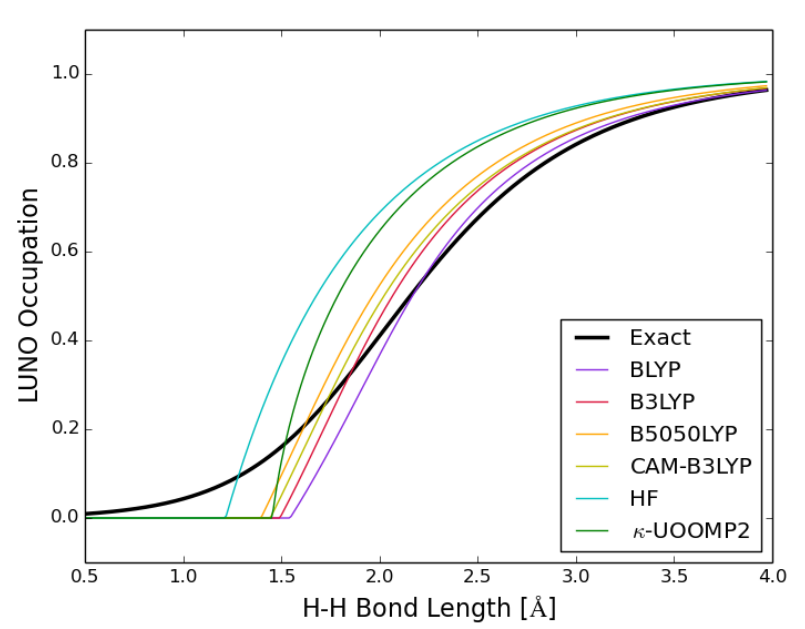

FIG. 1: LUNO occupation $\left(n_{L U N O}\right)$ vs internuclear distance for stretched $\mathrm{H}_{2}$. Exact and $\kappa$-UOOMP2 results were obtained with the aug-cc-pVQZ basis, while the DFT results were obtained from the aug-pc- 4 basis.

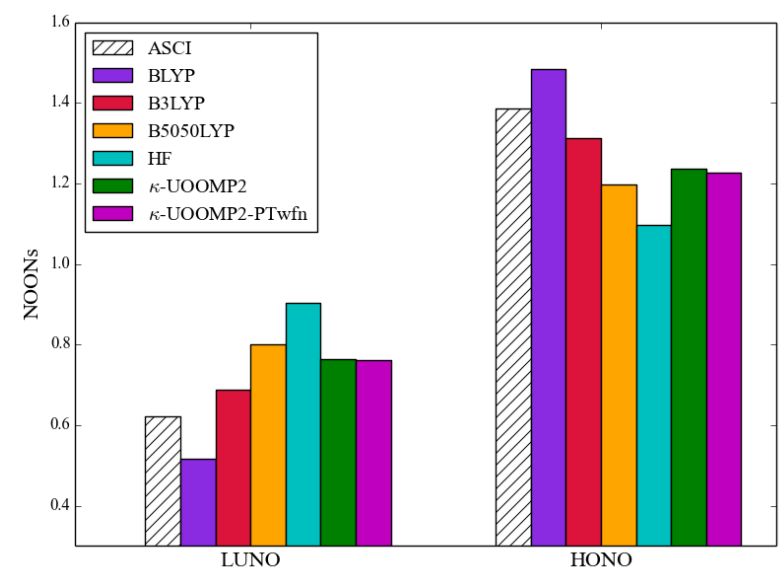

FIG. 2: A comparison of LUNO and HONO occupation numbers, computed with various methods, for para-benzyne.

$\kappa$-UOOMP2-PTwfn represents the values calculated from Eq. (6).

higher percentage of EXX into the functional form provides the nonlocal, orbital-dependent exchange necessary to describe the derivative discontinuity, and DE can thus be reduced by global hybrids with a larger amount of EXX (e.g. B5050LYP) or range-separated hybrid (RSH) functionals (e.g. CAM-B3LYP ${ }^{83}$ ). ${ }^{84,85}$ B5050LYP provides a substantial improvement over B3LYP, and CAMB3LYP further improves the predicted NOONs.

In the context of transition metal complexes, DE can have a dramatic effect on the predicted covalency or dative nature of metal-ligand interactions. It has been shown that calculated spin-densities, and derived electron-nuclear hyperfine coupling constants (which can be measured by EPR spectroscopy), are sensitive to DE, and can lead to extreme variations in predicted paramagnetic NMR shifts in the range of $\mathcal{O}(1000)$ ppm. ${ }^{86,87}$ For- 


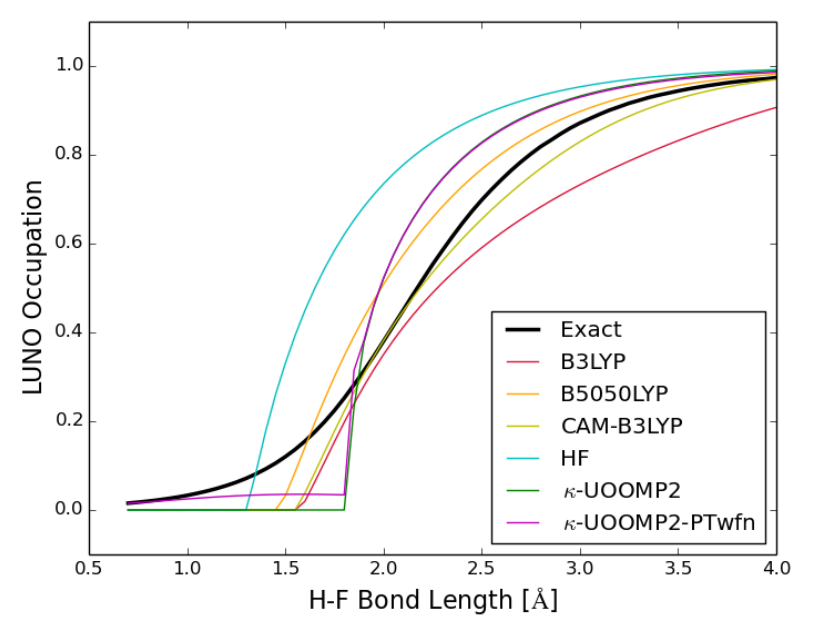

FIG. 3: LUNO occupation $\left(n_{L U N O}\right)$, as computed with various methods compared to the ASCI benchmark, vs internuclear distance for stretched hydrogen fluoride. $\kappa$-UOOMP2 results were obtained with the aug-cc-pVQZ basis, DFT results with the aug-pc- 4 basis. We show ASCI values with the cc-pVDZ basis, where $E_{P T 2}$ could be fully converged $\left(<10^{-3} \mathrm{Ha}\right)$. In the cc-pVTZ basis, we checked that $n_{L U N O}^{A S C I}$ deviated by at most 0.05 from the converged value in the cc-pVDZ basis.

tunately, the presence of DE can be diagnosed in straightforward ways, implied, e.g., by deviations from straightline behavior in plots of energy vs fractional occupation number ${ }^{64,87}$ (see Ref. 88 for this analysis of the fluoride anion relevant to dissociated hydrogen-fluoride). In such cases methods such as $\kappa$-UOOMP2, B5050LYP, and RSH functionals are to be preferred over pure or typical global hybrids such as BLYP or B3LYP, respectively.

Finally, we note that using the 1-particle density matrix from the $\kappa$-UOOMP2 wavefunction to compute the LUNO tracked the value obtained from using the $\kappa$ UOOMP2 orbitals in Eq. (5) nearly indistinguishably, save for at bond lengths shorter than the CF point where it is not constrained to zero as in the single-determinant methods.

\section{B. Metal hydride diatomics: spin and spatial symmetry breaking can imply multireference ground-states}

In this section we investigate the $3 \mathrm{~d}$ transition metal hydrides, and will glean insights into the connection between SC and symmetry breaking. These systems are small enough such that near-exact wavefunctions can be obtained, and we use ASCI to converge FCI-quality wavefunctions (with a Ne frozen-core for the metal atom). As mentioned in the Introduction, $3 \mathrm{~d}$ metal diatomics can possess surprisingly complicated electronic structures, resulting in a host of literature showing that SR methods (and some MR ones) have pronounced difficulty in predicting experimental thermochemistry. As will be discussed below, metal hydrides are linear molecules, point
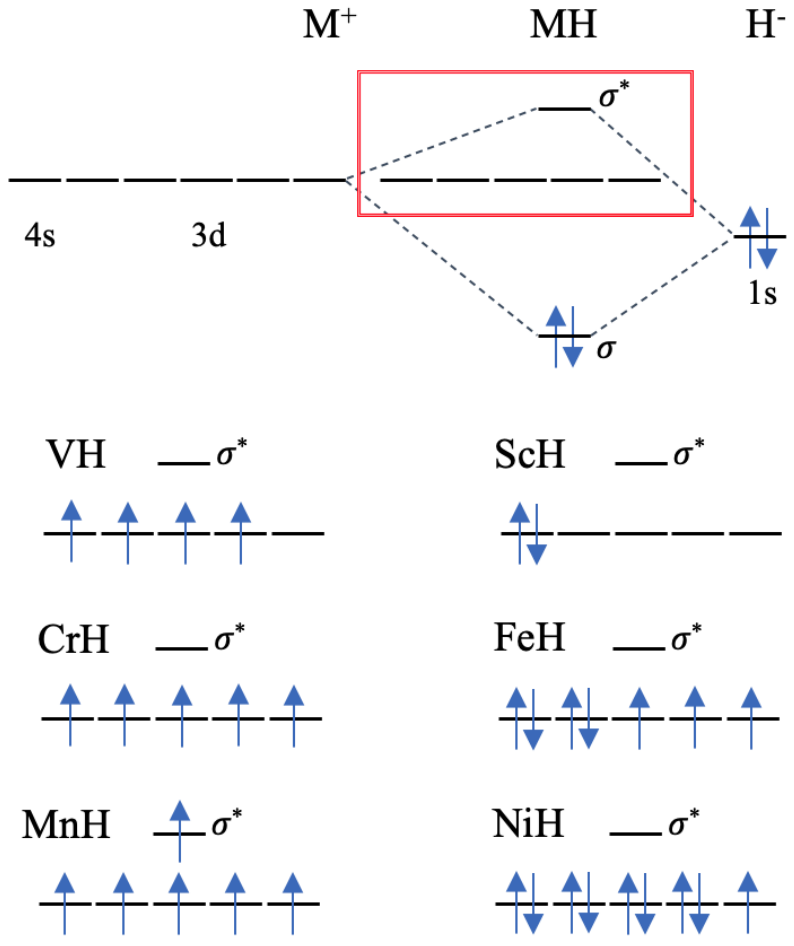

FIG. 4: Molecular orbital diagrams for metal-hydrides. Orbitals in the red block define the analog of 10Dq for diatomics.

group $\mathrm{C}_{\infty v}$, for which the Jahn-Teller theorem does not apply. Therefore, MR character can arise in the GS due to both spatial and spin symmetry requirements.

Fig. 4 shows a qualitative schematic of an expected molecular orbital (MO) diagram, for the metal hydride compounds. The shell of formally non-bonding orbitals (consisting of one $\sigma$, two $\pi$, and two $\delta$ orbitals) are, for now, treated as a single degenerate shell on the grounds of very small energetic splittings between the five orbitals. For our analysis of SSB, this will be adequate; however, a closer look is required for our analysis of spatial SB in $\mathrm{TiH}$ and $\mathrm{CoH}$.

Table I shows the $\left\langle S^{2}\right\rangle$ values from UHF, UDFT, $\kappa$ UOOMP2, and UOOCCD ${ }^{89}$, compared with the exact spin quantum number. Comparing with the MO diagrams in Fig. 4, the first thing to notice is that a number of the diatomics which do not show SSB are HS states (VH, $\mathrm{CrH}, \mathrm{MnH}$ ). HS states are known to be of SR nature. ${ }^{90} \mathrm{SSB}$ can only occur when there are paired valence electrons, as in the Fe- $\mathrm{Zn}$ hydrides. In addition, a higher spin-state must be sufficiently close in energy to mix into the unrestricted SR wavefunction of the LS state. In the single-particle/MO picture, this means that the lowest unoccupied molecular orbital (LUMO) in the dominant electronic configuration must be low-lying enough to be occupied (via spin-flip excitation) in the HS state. As will be discussed further in the next section, tetrahedral and octahedral metal complexes exhibit a splitting between $\mathrm{t}_{2 g}$ and $\mathrm{e}_{g}$ levels known as the 10Dq. 
Below we discuss the analogs of this for linear $3 \mathrm{~d}$ metal hydrides.

As shown in the red box of Fig. 4, the analog of 10Dq that can modulate $\mathrm{SSB}$ for $\mathrm{FeH}, \mathrm{CoH}, \mathrm{NiH}$, and $\mathrm{CuH}$ is the gap between the non-bonding $4 \mathrm{~s}$ and $3 \mathrm{~d}$ metal orbitals with the antibonding $\sigma^{*}$. The lowest excitation energies as (roughly) estimated via TDDFT within the TDA approximation (with the B3LYP functional and def2-SV(P) basis) for $\mathrm{CuH}$ and $\mathrm{ZnH}$ are 2.2 and $4.4 \mathrm{eV}$, respectively, suggesting that for these diatomics (though especially the latter) this gap is too large for HS states to compete. In contrast, the spin-contamination found in $\mathrm{FeH}, \mathrm{CoH}$, and $\mathrm{NiH}$ with all dynamically-correlated SR theories considered suggests that for these three species this gap is small enough such that SSB can occur. Going from $\mathrm{CuH}$ to $\mathrm{FeH}$, the fractional population of the $\sigma^{*}$ NO, as given by ASCI calculations and shown in Table II, monotonically increases from 0.06 to 0.35 . Correspondingly, the gap between the non-bonding manifold and the $\sigma^{*}$ orbital can be expected to decrease in this direction due to i) the decrease in metal atomic electronegativity which, in the MO picture, increases the energy of the metal AOs and, as a result, the metal non-bonding MOs relative to the $\sigma^{*}$ orbital (which has partial ligand character); and ii) the increase in metal atomic radius which, according to ligand field theory, should decrease the splitting of bonding and antibonding orbitals upon complexation with the hydride. As a result, the HS state formed by populating the $\sigma^{*}$ becomes increasingly more energetically-competitive with the LS state, resulting in proportional SSB.

For ScH, which is the only diatomic in the left-half of the row with significant post-HF SSB, the analog of the 10Dq parameter corresponds to the gap between the 4s and 3d MOs (0.4 eV with TDDFT/TDA). In other words, the intruding HS state responsible for the spin contamination is formed when the spin-down electron in the doubly-occupied 4s orbital undergoes a spin-flip excitation into the energetically proximate non-bonding $3 \mathrm{~d}$ shell. For ScH at the UOOCCD level of theory we note that computing $\left\langle S^{2}\right\rangle$ via Eq. 5 with the optimized orbitals led to a value of 1 , whereas using the full UOOCCD wavefunction yielded $\left\langle S^{2}\right\rangle=1.75$. The RooCCD energy is found to be lower than that of UOOCCD (but slightly higher than the ASCI energy), which reflects the fact that CC wavefunctions are highly sensitive to (and can be adversely affected by) spin-contamination in the reference state.

In light of the four half-occupied ASCI-computed NOONs shown in Table III, the lack of SSB in TiH is rather provocative. Indeed, due to the quartet multiplicity, there are no paired electrons in the 4s-3d MO manifold which would imply no SSB, which until now has implied a SR wavefunction. TiH's four NOONs of 0.5 can be explained in the context of spatial, rather than spin, SB as follows. The ${ }^{4} \Phi$ term symbol denotes a doubly-degenerate $(E)$ irreducible representation $\left({ }^{4} \Phi_{x}\right.$
$\mathrm{TiH}$
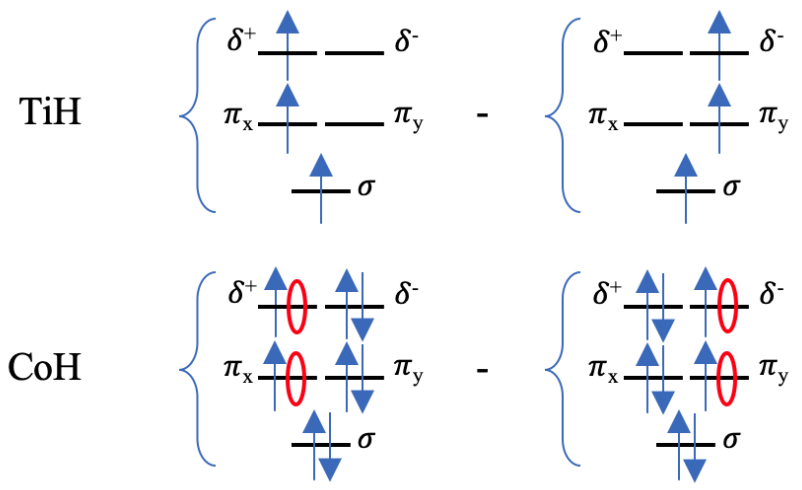

FIG. 5: Spatially symmetric ${ }^{4} \Phi_{x}$ and ${ }^{3} \Phi_{x}$ wavefunction schematics for $\mathrm{TiH}$ and $\mathrm{CoH}$, respectively.

and $\left.{ }^{4} \Phi_{y}\right)$ where, e.g., ${ }^{4} \Phi_{x}=\frac{1}{\sqrt{2}}\left(\left|\sigma \delta_{+} \pi_{x}\right\rangle-\left|\sigma \delta_{-} \pi_{y}\right\rangle\right)^{20}$. The corresponding electron configurations are illustrated in Fig. 5, where the wavefunction is a linear combination of two configurations varying in their occupation of the $\pi$ and $\delta$ orbitals. The term symbol of the wavefunction for $\mathrm{CoH}$ leads to an analogous situation but for two holes rather than two electrons, e.g. ${ }^{3} \Phi_{x}=\frac{1}{\sqrt{2}}\left(\left|\sigma^{2} \pi_{y}^{2} \delta_{-}^{2} \pi_{x} \delta_{+}\right\rangle-\left|\sigma^{2} \pi_{x}^{2} \delta_{+}^{2} \pi_{y} \delta_{-}\right\rangle\right),{ }^{20}$ also illustrated in Fig. 5. Thus, for $\mathrm{TiH}$ and $\mathrm{CoH}$ the four 0.5 and 1.5 NOONs, respectively, imply MR character due to spatial symmetry. This can occur in the absence of SSB $(\mathrm{TiH})$, or in addition to it $(\mathrm{CoH})$.

\section{Spin-symmetry breaking in metal complexes modulated by ligand position in the spectrochemical series}

For the rest of the paper, we turn to $3 \mathrm{~d}$ metal complexes with higher coordination numbers, for which JT distortions readily lift any degenerate electron configurations due to spatial symmetry. In this section we introduce the correspondence between SSB and the magnitude of the ligand-field paramater, 10Dq, which denotes the splitting between $\mathrm{t}_{2 g}$ and $\mathrm{e}_{g}$ orbitals for tetrahedral $\left(\mathrm{T}_{d}\right)$ and octahedral $\left(\mathrm{O}_{h}\right)$ complexes. Whether or not this can be interpreted as a marker for MR character will be postponed to the next section. Our discussion will center around $\mathrm{O}_{h}$ complexes, and in particular $\mathrm{Fe}(\mathrm{II}) \mathrm{L}_{6}$, which implies a $d^{6}$ configuration. In the $\mathrm{O}_{h}$ field, which yields a three below two d orbital ligand-field splitting, the LS state is a singlet with each of the three $t_{2 g}$ orbitals doubly occupied, whereas the HS state is a quintet formed by unpairing and spin-flipping two electrons from the $\mathrm{t}_{2 g}$ to the $\mathrm{e}_{g}$ orbitals. These states, along with the definition of 10Dq, are shown in Fig. 6. A small (large) $10 \mathrm{Dq}$ value generally results in the GS being HS (LS), respectively, though a complete analysis involves examining the delicate balance between i) the energetic cost to promote from the $\mathrm{t}_{2 g}$ to the $\mathrm{e}_{g}(10 \mathrm{Dq})$, ii) the ener- 
TABLE I: Diatomic species, term symbols, and exact and calculated $\left\langle S^{2}\right\rangle$ values.

\begin{tabular}{lrrrrrrr}
\hline \hline & $S_{\text {exact }}^{2}$ & $S_{U H F}^{2}$ & $S_{U B 3 Y P}^{2}$ & $S_{U C A M-B 3 L Y P}^{2}$ & $S_{U B 5050 L Y P}^{2}$ & $S_{\kappa U O O M P 2}^{2}$ & $S_{\text {UOOCCD }}^{2}$ \\
\hline $\mathrm{ScH}\left({ }^{1} \Sigma^{+}\right)$ & 0.00 & 1.00 & 0.60 & 0.58 & 0.54 & 1.00 & 1.00 \\
$\mathrm{TiH}\left({ }^{4} \Phi\right)$ & 3.75 & 3.75 & 3.76 & 3.76 & 3.75 & 3.75 & 3.75 \\
$\mathrm{VH}\left({ }^{5} \Delta\right)$ & 6.00 & 6.01 & 6.01 & 6.01 & 6.01 & 6.01 & 6.01 \\
$\mathrm{CrH}\left({ }^{6} \Sigma^{+}\right)$ & 8.75 & 8.87 & 8.79 & 8.79 & 8.80 & 8.82 & 8.79 \\
$\mathrm{MnH}\left({ }^{7} \Sigma^{+}\right)$ & 12.00 & 12.00 & 12.00 & 12.00 & 12.00 & 12.00 & 12.00 \\
$\mathrm{FeH}\left({ }^{4} \Delta\right)$ & 3.75 & 4.72 & 4.05 & 4.10 & 4.39 & 4.44 & 4.48 \\
$\mathrm{CoH}\left({ }^{3} \Phi\right)$ & 2.00 & 2.95 & 2.16 & 2.19 & 2.48 & 2.23 & 2.43 \\
$\mathrm{NiH}\left({ }^{2} \Delta\right)$ & 0.75 & 1.68 & 0.81 & 0.82 & 0.98 & 0.79 & 0.92 \\
$\mathrm{CuH}\left({ }^{1} \Sigma^{+}\right)$ & 0.00 & 0.00 & 0.00 & 0.00 & 0.00 & 0.00 & 0.00 \\
$\mathrm{ZnH}\left({ }^{2} \Sigma^{+}\right)$ & 0.75 & 0.76 & 0.75 & 0.76 & 0.76 & 0.76 & 0.76 \\
\hline \hline
\end{tabular}

TABLE II: Occupation number corresponding to the $\sigma^{*}$ natural orbital from ASCI/def2-SV(P) calculations, along with other MR diagnostics based on CCSD $(\mathrm{T}) /$ cc-pVTZ-DK from Ref. 31.

\begin{tabular}{|c|c|c|c|c|c|}
\hline & $\sigma^{*} \mathrm{NOON}^{\dagger}$ & $T_{1}$ & $D_{1}$ & $\left|t_{1}^{\max }\right|$ & $\%$ TAE \\
\hline $\mathrm{ScH}\left({ }^{1} \Sigma^{+}\right)$ & & 0.02 & 0.04 & 0.05 & 1.3 \\
\hline $\operatorname{TiH}\left({ }^{4} \Phi\right)$ & & 0.06 & 0.12 & 0.13 & 0.8 \\
\hline $\mathrm{VH}\left({ }^{5} \Delta\right)$ & & 0.09 & 0.21 & 0.22 & 0.2 \\
\hline $\mathrm{CrH}\left({ }^{6} \Sigma^{+}\right)$ & & 0.17 & 0.43 & 0.48 & 1.6 \\
\hline $\mathrm{MnH}\left({ }^{7} \Sigma^{+}\right)$ & & 0.02 & 0.05 & 0.05 & -1.6 \\
\hline $\mathrm{FeH}\left({ }^{4} \Delta\right)$ & 0.35 & 0.10 & 0.29 & 0.47 & 10.6 \\
\hline $\mathrm{CoH}\left({ }^{3} \Phi\right)$ & 0.22 & 0.07 & 0.21 & 0.22 & 12.4 \\
\hline $\mathrm{NiH}\left({ }^{2} \Delta\right)$ & 0.14 & 0.06 & 0.17 & 0.17 & 10.6 \\
\hline $\mathrm{CuH}\left({ }^{1} \Sigma^{+}\right)$ & 0.06 & 0.04 & 0.13 & 0.09 & 3.8 \\
\hline $\mathrm{ZnH}\left({ }^{2} \Sigma^{+}\right)$ & & 0.03 & 0.08 & 0.11 & -1.7 \\
\hline
\end{tabular}

$\dagger$ This work.

TABLE III: Frontier NOONs from ASCI/def2-SV(P) calculations for $\mathrm{TiH}$ and $\mathrm{CoH}$. The four NOONs in the central box correspond to $\pi$ and $\delta$ orbitals shown in Fig. 5.

\begin{tabular}{lll|llll|l}
$\mathrm{TiH}$ & 1.95 & 0.99 & 0.51 & 0.51 & 0.50 & 0.50 & 0.04
\end{tabular} \begin{tabular}{lll|llll|l}
$\mathrm{CoH}$ & 1.93 & 1.78 & 1.50 & 1.50 & 1.47 & 1.47 & 0.22 \\
\hline
\end{tabular}

getic cost to pair two opposite-spin electrons in the same $\mathrm{t}_{2 g}$ orbital, and iii) the stabilizing exchange interaction between same-spin electrons.

It is well-known that the 10Dq parameter can be modulated by a ligand's position on the spectrochemical series, which reflects whether the metal(M)-ligand(L) interaction is characterized by L-to-M $\sigma$ donation only, or, in addition, L-to-M $\pi$ donation or M-to-L $\pi$ backbonding. MO theory provides an intuitive model which corroborates the experimentally-determined trend that $10 \mathrm{Dq}$ gets smaller going from $\pi$-accepting to $\sigma$-donating-only to $\pi$-donating ligands. ${ }^{91}$

Table IV shows calculated $\left\langle S^{2}\right\rangle$ values at the UHF,
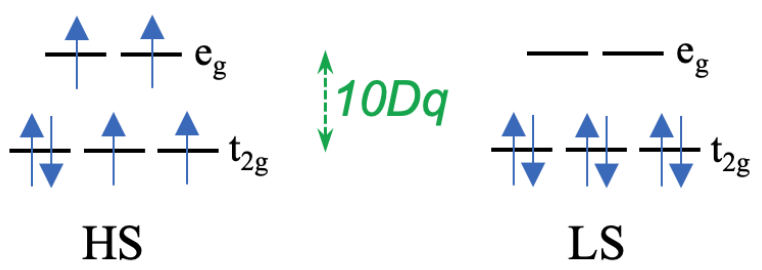

FIG. 6: High-spin (HS) And low-spin (LS) electron configurations corresponding to $\mathrm{d}^{6} \mathrm{Fe}(\mathrm{II}) \mathrm{X}_{6}$ complexes.

UB3LYP, UCAM-B3LYP, UB5050LYP, and $\kappa$-UOOMP2 levels of theory for $\mathrm{O}_{h} \mathrm{Fe}(\mathrm{II})$ complexes in the LS state representing a range of ligand-field strengths. The CO ligand is perhaps the strongest-field $\pi$-acceptor in the spectrochemical series, and there is no SSB at any level of theory (not even UHF). We attribute this to the 10Dq being sufficiently large such that the HS state is energetically inaccessibile and therefore unable to mix into the LS wavefunction. This implies that the LS state is SR with very strong-field $/ \pi$-accepting ligands (indeed, as will be argued in the final section, the inaccuracy of MP2-based methods for organometallic thermochemistry is due to other reasons). As the ligand-field strength is attenuated $\left(\mathrm{NH}_{3}\right.$ and $\mathrm{H}_{2} \mathrm{O}$ are $\sigma$-donation only ligands; the halides $\pi$-donor ligands), the magnitude of $10 \mathrm{Dq}$ decreases, and the increasing accessibility of the HS configuration manifests as deviations from exact $\left\langle S^{2}\right\rangle$ values in the LS state.

The trend of increasing SSB going toward weak-field ligands along the spectrochemical series is present in all methods investigated. In fact, all DFT and $\kappa$-UOOMP2 methods yield qualitatively similar values of $\left\langle S^{2}\right\rangle$ (with the exception of the artificial SSB from the B5050LYP functional for $\mathrm{Fe}\left(\mathrm{NH}_{3}\right)_{6}^{2+}$, which, as might be expected, is nearly half the value found from UHF). We have used a polarizable continuum model $(\epsilon=78.4)$ for the DFT calculations of all anionic species, as motivated by the interesting case of the complex with fluoride $\left(\mathrm{F}^{-}\right)$ligands: In the gas phase, the $\left\langle S^{2}\right\rangle$ value from B3LYP (1.74) exceeds that from UHF (1.49). The fluorine anion is known to have a positive HOMO eigenvalue when employing 
TABLE IV: Calculated $\left\langle S^{2}\right\rangle$ values of the LS state of $\mathrm{Fe}(\mathrm{II}) \mathrm{X}_{6}^{2+/ 4-}$. All DFT calculations of anions (i.e. complexes with halide ligands) use the C-PCM polarizable continuum model, with dielectric constant 78.4 and a van der Waals radius of $2 \AA$ for Fe. 10Dq estimates obtained from Ref. 92 via the difference in CASPT2 energies of the ${ }^{5} T\left(t_{2 g}^{4} e_{g}^{* 2}\right)$ and ${ }^{5} E\left(t_{2 g}^{3} e_{g}^{* 3}\right)$ states.

\begin{tabular}{|c|c|c|c|c|c|c|c|}
\hline & $S_{\text {exact }}^{2}$ & $S_{U H F}^{2}$ & $S_{U B 3 L Y P}^{2}$ & $S_{U C A M-B 3 L Y P}^{2}$ & $S_{U B 5050 L Y P}^{2}$ & $S_{\kappa U O O M P 2}^{2}$ & $10 \mathrm{Dq}[\mathrm{eV}]$ \\
\hline \multicolumn{8}{|l|}{$\underline{\mathrm{LS}}$} \\
\hline $\mathrm{Fe}(\mathrm{CO})_{6}^{2+}$ & 0 & 0.00 & 0.00 & 0.00 & 0.00 & 0.00 & 4.90 \\
\hline $\mathrm{Fe}(\mathrm{NCH})_{6}^{2+}$ & 0 & 0.44 & 0.00 & 0.00 & 0.00 & 0.00 & 3.21 \\
\hline $\mathrm{Fe}\left(\mathrm{NH}_{3}\right)_{6}^{2+}$ & 0 & 1.01 & 0.00 & 0.05 & 0.45 & 0.00 & 2.69 \\
\hline $\mathrm{Fe}\left(\mathrm{H}_{2} \mathrm{O}\right)_{6}^{2+}$ & 0 & 1.36 & 0.86 & 0.90 & 1.08 & 0.68 & 1.64 \\
\hline $\mathrm{FeF}_{6}^{4-}$ & 0 & 1.49 & 1.03 & 1.07 & 1.23 & 1.04 & \\
\hline $\mathrm{FeCl}_{6}^{4-}$ & 0 & 1.71 & 1.40 & 1.42 & 1.53 & 1.41 & \\
\hline $\mathrm{FeBr}_{6}^{4-}$ & 0 & 1.74 & 1.43 & 1.46 & 1.58 & 1.41 & \\
\hline
\end{tabular}

functionals such as B3LYP in all but exceedingly large basis sets ${ }^{93}$, and the curvature in a plot of the energy as a function of fractional occupation number is a hallmark of DE, which further encourages long tails in the radial charge density. ${ }^{88,94}$. Indeed, for $\mathrm{FeF}_{6}^{4-}$ in the gasphase with B3LYP, 21 of the 42 occupied Kohn-Sham orbital eigenvalues were greater than zero! When improving (slightly) the description of a continuum orbital via the def2-SVPD basis set, $\left\langle S^{2}\right\rangle$ is further increased to 1.94, which reflects the expected narrowing of the singlettriplet gap as continuum orbitals become more involved. Using a dielectric characteristic of water solvent, only the HOMO eigenvalue remained positive with a value of 0.01 , and the resulting $\left\langle S^{2}\right\rangle$ decreased from 1.74 to 1.03 , now below the UHF value and in agreement with functionals with more EXX (and thus less DE) and $\kappa$-UOOMP2, which is free of DE. We note that increasing $\epsilon$ and the flexibility of the basis set can make all occupied B3LYP eigenvalues negative, but we did not find any large effect on the SSB behavior (e.g., a HOMO eigenvalue of -0.11 is obtained with $\epsilon=1000$ and the def2-SVPD basis, and $\left.\left\langle S^{2}\right\rangle=1.20\right)$.

\section{Does SSB imply SC or variational collapse?}

Many of these $\mathrm{O}_{h} \mathrm{Fe}(\mathrm{II})$ compounds are, in fact, prototypical and well-studied spin-crossover complexes (SCC). Under appropriate external conditions, e.g. pressure or protein environment, SCCs can exhibit transitions between LS and HS states, enabling the precise control of interesting magnetic phenomena. ${ }^{95-98}$ Four of the $\mathrm{Fe}(\mathrm{II})$ complexes - $\left[\mathrm{Fe}\left(\mathrm{H}_{2} \mathrm{O}\right)_{6}\right]^{2+},\left[\mathrm{Fe}\left(\mathrm{NH}_{3}\right)_{6}\right]^{2+},\left[\mathrm{Fe}(\mathrm{NCH})_{6}\right]^{2+}$, and $\left[\mathrm{Fe}(\mathrm{CO})_{6}\right]^{2+}$ - have received significant attention from theoreticians utilizing an array of sophisticated $a b$ initio methods (in the absence of gas-phase experimental measurements). ${ }^{99}$ Spin gaps pose a difficult problem for DFT methods, since a range of splittings can be obtained depending on the functional employed (and in particular, for global hybrids, the amount of EXX incorporated). ${ }^{99,100}$

A notable study of these four Fe(II) potential SCCs compared the results of a host of DFT functionals and wavefunction methods with those from diffusion Monte Carlo (DMC) calculations (employing pseudopotentials and within the fixed-node approximation). ${ }^{101} \mathrm{It}$ is claimed therein that the $\mathrm{CO}$ species is markedly multiconfigurational. In another study, all-electron DMC calculations have been performed. For the complex with the CO ligand, the calculated spin gaps with singledeterminant and multi-determinant trial wavefunctions agreed to within the $0.005 \mathrm{Ha}$ error bar, suggesting that this complex is not strongly correlated. ${ }^{100}$ Neese and coworkers did not consider the $\mathrm{CO}$ species but carried out a detailed investigation of the remaining three $\mathrm{Fe}(\mathrm{II})$ complexes with DLPNO-CCSD(T) methods employing large basis sets. ${ }^{102}$

Now, we will add to the data in Table IV the finding that all HS states of the four Fe(II) complexes presently under consideration did not exhibit any SSB. This observation, found too for the diatomic species above, is quite general, and provides the point of departure for the development of spin-flip approaches to, e.g., TDDFT and wavefunction meethods (CI, CC, and more). This can be understood in the context of the quantum theory of angular momentum, where a high spin quantum number can have numerous, e.g., $m_{s}$ states, at least one of which can typically be well-described by a single determinant. For the LS singlet species, going from $\mathrm{CO}, \mathrm{NCH}, \mathrm{NH}_{3}$, to $\mathrm{H}_{2} \mathrm{O}$ (i.e. going from strong-field $/ \pi$-accepting toward weak-field $/ \sigma$-donation-only) we find that the deviation of $\left\langle S^{2}\right\rangle_{U H F}$ increases consistently. UB3LYP completely restores the SSB found in UHF, except in the case of $\mathrm{Fe}\left(\mathrm{H}_{2} \mathrm{O}\right)_{6}^{2+} . \quad \kappa$-UOOMP2 yields similar conclusions. In disagreement with the aforementioned claims from other groups in the literature, our results strongly suggest that the CO species is not MR, given that no SSB occurs even at the UHF level. On the other hand, all dynamically correlated methods here suggest that the $\mathrm{LS} \mathrm{H}_{2} \mathrm{O}$ species has HS character mixed in. This SSB behavior is consistent with calculated $10 \mathrm{Dq}$ values from Ref. 92, reproduced in Table IV.

The adiabatic LS/HS gaps, i.e. derived from separately optimized LS and HS geometries, have been calcu- 
TABLE V: Vertical energy difference $[\mathrm{eV}]$ between the LS state, and the HS state in LS geometry. A negative value means the LS state is more stable. UHF, UB3LYP, and $\kappa$-UOOMP2 predictions are compared with reference values from $\operatorname{CCSD}(\mathrm{T})$, all in the $\operatorname{def} 2-\mathrm{SV}(\mathrm{P})$ basis set.

\begin{tabular}{lllll}
\hline \hline & CCSD $(\mathrm{T})^{\dagger}$ & UHF & UB3LYP & $\kappa$-UOOMP2 \\
\hline $\mathrm{Fe}(\mathrm{CO})_{6}{ }^{2+}$ & -3.73 & -1.15 & -4.48 & -6.79 \\
$\mathrm{Fe}(\mathrm{NCH})_{6}{ }^{2+}$ & -0.26 & 1.34 & -1.20 & -0.83 \\
$\mathrm{Fe}\left(\mathrm{NH}_{3}\right)_{6}{ }^{2+}$ & 0.87 & 1.98 & -0.11 & 0.70 \\
$\mathrm{Fe}\left(\mathrm{H}_{2} \mathrm{O}\right)_{6}{ }^{2+}$ & 1.83 & 2.41 & 0.78 & 1.78 \\
\hline \hline
\end{tabular}

${ }^{\dagger} \mathrm{R}$ for singlet, $\mathrm{U}$ for quintet (the latter, in all cases, is essentially spin-pure)

lated in Refs. 102 and 92. Both CASPT2 and CCSD(T) methods predict that only $\mathrm{Fe}(\mathrm{CO})_{6}^{2+}$ has a LS GS (due to the large $10 \mathrm{Dq}$ of $4.9 \mathrm{eV}$ ), while the GSs of the three other molecules with weaker-field ligands are HS. Evidently, the $\mathrm{NCH}$ ligand reduces the 10Dq such that the cost of promoting two electrons to the $\mathrm{e}_{g}$ orbitals is more than compensated by the stabilization provided by exchange (with four spin-up, unpaired electrons) and by unpairing two pairs that had been in the $\mathrm{t}_{2 g}$ manifold. $\mathrm{NH}_{3}$ and $\mathrm{H}_{2} \mathrm{O}$ ligands continue the trend of reducing 10Dq, and, of the three factors mentioned previously, the HS state drops lower in energy due to the increasing ease of promoting from $\mathrm{t}_{2 g}$ to $\mathrm{e}_{g}$.

Yet while the adiabatic energy difference is relevant for determining the proper GS multiplicity when calculating thermochemical properties, it is the vertical gap - i.e. the difference between the LS state and the HS state in the LS geometry - which is directly related to SSB behavior: At a fixed geometry, when the HS state is the GS, SSB in the LS state (assuming it is a saddle point in the energy surface in orbital space) is to be expected from unrestricted variational methods, wherein constraining a molecule's multiplicity is done by constraining the projection $S_{z}$ (equal to $N_{\alpha}-N_{\beta}$ ) rather than the value of $S^{2}$. As a consequence, such methods will include suitable HS contributions into the single-determinant reference in order to minimize its energy. However, the presence of HS contributions at the single determinant level for such species does not necessarily imply that the LS state has significant MR character. Indeed, with a large-enough LS-HS gap, it is quite possible that the lowest LS state is dominated by a single determinant, while being above the HS state in energy. UHF/UKS calculations for such LS states can still exhibit SSB due to inclusion of HS character, despite the actual state being fairly SR (as indicated by NOONs). A classic main-group example of this is $\mathrm{CH}_{2}$, where the triplet state is the GS while the lowest singlet state is predominantly closed-shell (HONO occupation of 1.89 and LUNO occupation of 0.09$)$. UHF/UKS calculations on $S_{z}=0 \mathrm{CH}_{2}$ would generate $\mathrm{SSB}$, as it is energetically favorable to contaminate the closed-shell singlet with the lower energy triplet. Several species in Table V provide additional examples, as described later.
Table $\mathrm{V}$ reveals that, at the LS geometry, UHF puts the HS state energetically below the LS state in all cases except for the carbonyl complex, which may explain why only the carbonyl complex was spin-pure at this level of theory (Table IV). UB3LYP and $\kappa$-UOOMP2 predictions of the relative spin-state ordering for the $\mathrm{NCH}$ complex are in agreement with the $\operatorname{CCSD}(\mathrm{T})$ reference, which suggests that UHF has overstabilized the HS state (a consequence of HF's neglect of all correlation except for same-spin exchange stabilization) such that its predicted state ordering is incorrect. Going toward weak-field ligands along the spectrochemical series, UB3LYP predicts a LS-below-HS ordering for the $\mathrm{CO}, \mathrm{NCH}$, and $\mathrm{NH}_{3}$ complexes, but the ordering switches for $\mathrm{Fe}\left(\mathrm{H}_{2} \mathrm{O}\right)_{6}^{2+}$. This can explain the onset of SSB in the UB3LYP level of theory at this molecule. Interestingly, the spin-state ordering predicted by $\kappa$-UOOMP2 switches from a LS to HS GS at the $\mathrm{NH}_{3}$ complex, however there is no SSB in Table IV; furthermore, we note that the vertical spingap at this level of theory deviates by $3 \mathrm{eV}$ from the CCSD(T) benchmark for the CO species - a clue which will become relevant in our later discussion of the possible inappropriateness of MP2 in describing interactions such as metal-carbonyl bonds. On the whole, it appears that with a HS GS, SSB in the LS excited-state need not imply MR character, but rather what we will refer to as "variational collapse." Indeed this is the reason that some sort of spin-projection ${ }^{53,103,104}$ is mandatory in such cases.

Consider $\mathrm{Fe}\left(\mathrm{H}_{2} \mathrm{O}\right)_{6}^{2+}$, for which SSB persists not only at the UHF level but at all DFT and $\kappa$-UOOMP2 theories. A CASSCF calculation with 6 electrons in 5 metal d orbitals yields NOONs of $1.960,1.954,1.954,0.066$, and 0.066. The small LUNO (0.066), in the context of the above study of the metal hydride diatomics, suggests SR character (though we note cautiously that CASSCF with 5 active orbitals is not the same as FCI in the full orbital space), and thus it seems that SSB simply reflects the HS-below-LS relative energetics. For $\mathrm{FeX}_{6}^{4-}$, with $\mathrm{X}=$ $\mathrm{F}, \mathrm{Cl}, \mathrm{Br}$ proceeding toward weaker-field $/ \pi$-donating ligands, the expected $10 \mathrm{Dq}$ decrease is a small effect, with LUNO populations resulting from minimal (e.g. 6e5o) CASSCF calculations of $0.076,0.100$, and 0.105 , respectively. For $\mathrm{FeF}_{6}^{4-}$ we verified that using a larger active space of $6 \mathrm{e} 15 \mathrm{o}$ to include a second d shell yielded a similar LUNO of 0.070 (vs 0.076 from 6e5o). The LS state of $\mathrm{Fe}(\mathrm{II}) \mathrm{Br}_{6}^{4-}$ is less $\mathrm{MR}$ than $\mathrm{NiH}, \mathrm{CoH}$, and $\mathrm{FeH}$ (with LUNO occupations of $0.14,0.22$, and 0.35 , respectively), and its LUNO value is strikingly similar to that of the lowest singlet state of $\mathrm{CH}_{2}(0.09)$, which is also predominately closed shell. This analysis suggests that neither the $\mathrm{CO}$ nor $\mathrm{H}_{2} \mathrm{O} \mathrm{LS} \mathrm{Fe}(\mathrm{II})$ complexes are MR, in agreement with previously reported D1 diagnostic values of 0.14 and 0.06 , which are below the 0.15 threshold suggested by Wilson and coworkers suggested for transition metals. ${ }^{31}$ Rather, the SSB observed from theories that include dynamic correlation is a manifestation of variational collapse. We thus reiterate that for excited-states, SSB should be used together with NOONs from a MR 
theory in order to probe for the presence of MR character.

To summarize, this set of $\mathrm{Fe}(\mathrm{II})$ complexes suggests that strong-field ligands such as CO (and, e.g. CN) yield single-configurational, LS GSs (which is also the case for $4 \mathrm{~d}$ and $5 \mathrm{~d}$ metal complexes, for the same reason - i.e. due to the large $10 \mathrm{Dq}$ that results from the strong M-L interaction). Weaker-field ligands on the spectrochemical series (e.g. $\mathrm{NH}_{3}, \mathrm{H}_{2} \mathrm{O}$ ) favor $\mathrm{HS}$ GSs due to the attenuation of $10 \mathrm{Dq}$ and the stabilizing exchange interactions among same-spin electrons (indeed, as the 10Dq of $\mathrm{T}_{d}$ compounds is roughly half of that of $\mathrm{O}_{h}$ compounds, the former in general have HS GSs). Practically speaking, for the calculation of thermochemical properties (for which only the GSs are relevant) of these types of coordination complexes, SSB will therefore be the exception rather than the rule, and encountering MR character need not be much of a concern (though appropriate SR methods must still be chosen with care, vide infra).

\section{E. Identifying MR character from spin-symmetry breaking}

We now seek to uncover chemical themes or circumstances, in addition to the diatomics analyzed above, in which static correlation can arise in the GS of transition metal complexes. Such conditions, while admittedly quite rare, are found in LS metal compounds which have very weak M-L bonding (encountered, e.g., in the gas phase in particular for $\sigma$-donating only ligands with low metal oxidation states), or antiferromagnetically-coupled spins occupying either separate metal centers or a metal center and a low-lying $\pi^{*}$ orbital of a redox-noninnocent ligand.

\section{Very weak metal-ligand bonding}

In Ref. 34 the ligand dissociation energies of 34 metal complexes, formed via ligand coordination of neutral or cationic $3 \mathrm{~d}$ metals in the gas-phase, were investigated. We have computed $\left\langle S^{2}\right\rangle$ with respect to UHF, UB3LYP, and $\kappa$-UOOMP2 orbitals for all GSs involved. Table VI shows the cases for which $\mathrm{SSB}$ at the UHF level was restored in both UB3LYP and $\kappa$-UOOMP2 cases; Table VII shows those cases for which SSB persisted at the UB3LYP and $\kappa$-UOOMP2 levels.

We observe that the molecules in the former group, for which UHF SSB is restored with UB3LYP and $\kappa$ UOOMP2, consist of metals with CO ligands, except in one case (which will be discussed after). CO ligands can participate in both $\sigma$-donation and $\pi$-backbonding. The latter substantially strengthens the metal-ligand bond, increasing the $10 \mathrm{Dq}$ which decreases the chances of finding a truly strongly correlated state; hence, the spinsymmetry restoration when dynamic correlation is included via DFT or $\kappa$-UOOMP2.
TABLE VI: Cases in which spin symmetry breaking at the UHF level is restored with UB3LYP and $\kappa$-UOOMP2.

\begin{tabular}{lllll}
\hline \hline & $S_{\text {exact }}^{2}$ & $S_{U H F}^{2}$ & $S_{U B 3 L Y P}^{2}$ & $S_{\kappa U O O M P 2}^{2}$ \\
\hline $\mathrm{Co}\left(\mathrm{NH}_{3}\right)_{3}^{+}$ & 2 & 2.78 & 2.03 & 2.03 \\
$\mathrm{Cr}(\mathrm{CO})_{5}\left(\mathrm{H}_{2}\right)$ & 0 & 0.70 & 0.00 & 0.00 \\
$\mathrm{Cr}(\mathrm{CO})_{5}$ & 0 & 0.74 & 0.00 & 0.00 \\
$\mathrm{~V}(\mathrm{CO})_{5}^{+}$ & 2 & 2.35 & 2.03 & 2.02 \\
$\mathrm{~V}(\mathrm{CO})_{6}^{+}$ & 2 & 2.32 & 2.02 & 2.02 \\
\hline \hline
\end{tabular}

TABLE VII: Cases in which spin symmetry breaking at the UHF level persists in UB3LYP and $\kappa$-UOOMP2.

\begin{tabular}{|c|c|c|c|c|}
\hline & $S_{\text {exact }}^{2}$ & $S_{U H F}^{2}$ & $S_{U B 3 L Y P}^{2}$ & $S_{\kappa U O O M P 2}^{2}$ \\
\hline $\mathrm{Fe}\left(\mathrm{CH}_{2} \mathrm{O}\right)_{3}^{+}$ & 3.75 & 4.74 & 3.96 & 3.86 \\
\hline $\mathrm{Fe}\left(\mathrm{CH}_{2} \mathrm{O}\right)_{4}^{+}$ & 3.75 & 4.75 & 4.11 & 3.95 \\
\hline $\mathrm{Fe}\left(\mathrm{H}_{2} \mathrm{O}\right)_{3}^{+}$ & 3.75 & 4.55 & 3.89 & 3.95 \\
\hline $\mathrm{Fe}\left(\mathrm{H}_{2} \mathrm{O}\right)_{4}^{+}$ & 3.75 & 4.59 & 3.86 & 3.89 \\
\hline $\mathrm{Fe}\left(\mathrm{NH}_{3}\right)_{3}^{+}$ & 3.75 & 4.54 & 3.86 & 3.90 \\
\hline $\mathrm{Fe}\left(\mathrm{NH}_{3}\right)_{4}^{+}$ & 3.75 & 3.78 & 3.94 & 3.92 \\
\hline $\mathrm{Mn}\left(\mathrm{NH}_{3}\right)_{3}^{+}$ & 6 & 6.86 & 6.30 & 6.62 \\
\hline $\mathrm{Mn}\left(\mathrm{NH}_{3}\right)_{4}^{+}$ & 6 & 7.00 & 6.71 & 7.00 \\
\hline
\end{tabular}

The molecules in the second group (Table VII), for which SSB persists with UB3LYP and $\kappa$-UOOMP2, all have weak-field ligands which predominately participate in $\sigma$-donation, i.e. without $\pi$ back-donation. This is obvious for $\mathrm{H}_{2} \mathrm{O}$ and $\mathrm{NH}_{3}$ ligands. The $\mathrm{CH}_{2} \mathrm{O}$ ligand has a double bond between $\mathrm{C}$ and $\mathrm{O}$ atoms and coordinates at the $\mathrm{O}$ end, with $\mathrm{sp}^{2}$ hybridation that is, in principle, capable of $\pi$ interactions with the metal. We performed an EDA calculation which shows that while the formaldehyde ligand can $\pi$ accept, the backbonding charge-transfer component is less than half that found for the CO complex (see Table S2). The weaker $\pi$ backbonding to $\mathrm{CH}_{2} \mathrm{O}$ vs carbonyl appears to create a $10 \mathrm{Dq}$ comparable to $\sigma$-only ligands which enables SSB.

We also note that this second group of complexes contains only $\mathrm{Fe}$ and $\mathrm{Mn}$ ions. $\mathrm{Mn}^{+}$and $\mathrm{Fe}^{+}$are unique in that they have $4 \mathrm{~s}^{1} 3 \mathrm{~d}^{n-1}$ electronic configurations. When a Lewis base donates two electrons, one must go into a bonding orbital while the other into an antibonding orbital, which lowers the bond order and weakens the covalent bond more so than if the metal atom was $3 \mathrm{~d}^{n}$. As implied by bond strength trends with $\mathrm{H}_{2}$ and $\mathrm{CO}$ ligands, $\mathrm{Fe}^{+}$and $\mathrm{Mn}^{+}$require 24 and $113 \mathrm{~kJ} / \mathrm{mol}$, respectively, to promote the 4 s electron to attain the $3 \mathrm{~d}^{n}$ configuration. ${ }^{35}$ This trend is consistent with our finding that the SSB is more severe in the Mn vs the Fe ammonia species; indeed, the Mn molecule is the only species which has SSB with UB3LYP orbitals in excess of $10 \%$.

A parallel can be drawn between the chemical situation that occurs in these very weakly bound metal com- 
plexes and that which occurs while stretching bonds. Taking $\mathrm{H}_{2}$ as an example, the gap between the bonding and antibonding MOs decreases as the bond length is stretched; after a certain distance, the near-degeneracy of the orbitals (or equivalently, of the many-body singlet and triplet states) results in an unpairing of the closed-shell singlet state into a two-configurational, biradicaloid singlet wavefunction. When the wavefunction is constrained to a single-determinant with unrestricted orbitals, beyond the CF point the broken-symmetry determinant, $|\uparrow \downarrow\rangle$, acquires partial triplet character since $\left|S^{2}=2, M_{S}=0\right\rangle=\frac{1}{\sqrt{2}}(|\uparrow \downarrow\rangle+|\downarrow \uparrow\rangle)$. In these transition metal complexes, we generalize the two involved multiplicities to LS and HS (quartet and sextet for the Fe complexes, quintet and septet for the Mn complex), and the accessibility of the HS state due to the factors mentioned above can be visualized as a vertical compression of the entire MO diagram.

For septet $\mathrm{Mn}\left(\mathrm{NH}_{3}\right)_{4}^{+}$, the spin-up HOMO has predominately s character (as given by partial Mulliken populations from the B3LYP calculation). This suggests that, at least for this case, the fractional closed-shell unpairing corresponds to population of an orbital not in the $\mathrm{t}_{2 g}$ or $\mathrm{e}_{g}$ manifolds, but rather one derived from the $4 \mathrm{~s}$ metal AO. Indeed, the NOONs from a CASSCF(6e6o) calculation of the quintet have occupation numbers 1.06, $1,1,1,1,0.94$; the orbitals corresponding to the 1.06 and 0.94 NOONs have equal contributions from Mn s and d orbitals. The weak bonding apparently compresses the MOs such that the $4 \mathrm{~s}$ orbital, which is in typical cases well-separated and energetically-above the $3 \mathrm{~d}$ MOs, is close enough to the $3 \mathrm{~d}$ levels to stabilize the septet (via extra exchange). Indeed, $\operatorname{CASSCF}(6 \mathrm{e} 6 \mathrm{o}$ ) (and also $\kappa$ UOOMP2) predicts a septet GS with the quintet $\sim 5 \mathrm{mHa}$ higher in energy, and thus the quintet NOONs reflecting 6 unpaired electrons suggest that, rather than diagnosing an MR quintet GS, what we have witnessed instead is a variationally-driven collapse towards the HS state. This underscores the danger of using small active spaces in CASSCF methods, namely that the spin-state ordering can be in error without dynamic correlation, which can lead to a false diagnosis of MR character. However, even theories which do formally include dynamic correlation lead to inconclusive results: in the def2-TZVPP basis and with the DKH Hamiltonian, BP86 (pure) and B3LYP (global hybrid with $20 \%$ EXX) functionals put the quintet below the septet state, while PBE0 (global hybrid with $25 \%$ EXX) and HF reverse the ordering. These multiplicities are clearly very close in energy, and the possibility of a septet GS - at odds with conventional chemical intuition - is currently being re-investigated carefully with a number of $a b$ initio methods.

Finally, we comment that in contrast to the SSB found in $\mathrm{Mn}$ and Fe complexes with ammonia ligands discussed above, in $\mathrm{Co}\left(\mathrm{NH}_{3}\right)_{3}^{+} \mathrm{UHF}$ SSB is artificial (i.e. restored via UB3LYP and $\kappa$-UOOMP2) presumably because the $3 \mathrm{~d}^{8}$ configuration of Co, without s occupancy, enables relatively stronger bonding / ligand-field splitting even in the absence of $\pi$-backdonation.

While we were unable to perfectly correlate deviations in the calculated ligand-dissociation energies using SR methods such as DLPNO-CCSD(T) or DFT vs experiments with SSB at the UB3LYP or $\kappa$-UOOMP2 levels of theory, we note that the largest error from DLPNO$\mathrm{CCSD}(\mathrm{T})$ vs experiment among the 34 molecules investigated in Ref. 34 was for the $\mathrm{Fe}\left(\mathrm{NH}_{3}\right)_{4}^{+}$species $(9.15$ $\mathrm{kcal} / \mathrm{mol})$. For the $\mathrm{Mn}\left(\mathrm{NH}_{3}\right)_{4}^{+}$complex, the B3LYP, B97, M06, PBE0, and $\omega$ B97X-V functionals, along with DLPNO-CCSD $(\mathrm{T})$, consistently underestimated the experimentally measured ligand-dissociation energy in the range of $4.74-6.88 \mathrm{kcal} / \mathrm{mol}$ (though use of the septet multiplicity is being explored). We must point out, here, that such weakly-bound ions are unlikely to be found in solution. The binding energies are very weak, e.g. $10.0 \pm 1.7$ and $8.6 \pm 1.4 \mathrm{kcal} / \mathrm{mol}$ for Fe and Mn tetraammonium species, respectively, and in polar solvents such as water these complexes may readily dissociate. It is also likely that the $\mathrm{Mn}(\mathrm{I})$ and $\mathrm{Fe}(\mathrm{I})$ states will undergo redox events with the solvent to become the more stable $\mathrm{Mn}(\mathrm{II})$ and $\mathrm{Fe}(\mathrm{II})$ oxidation states.

\section{Redox-active complexes with non-innocent ligands}

The $a b$ initio prediction of electrochemical redox potentials of transition metal catalysts is a long-sought goal. Encouragingly, previous analyses of the deviations of DFT predictions from experimental measurements have revealed some systematic trends, ${ }^{105}$ and there are indications that computing the potential of the reference redox couple can encourage favorable error cancellation. ${ }^{106}$ However, one can easily find a sizable number of large outliers, and as the presence of MR character in the highly reduced species is one factor which would lead to erroneous predictions, the ability to quickly diagnose these situations is a prerequisite if computational predictions are to be reliable and predictive.

When an electrode reduces a homogeneous metal catalyst one or more times, making it redox-active toward substrates such as $\mathrm{CO}_{2}$ and $\mathrm{O}_{2}$, the reduction is most often metal-centered. ${ }^{107-111}$ Indeed, a unique property of transition metals is that they can accommodate multiple oxidation states, which can enable remarkable reactivity. However, large delocalized ligands can have low-lying $\pi^{*}$ orbitals which can be preferentially reduced vs a virtual metal d orbital. ${ }^{112-121}$ Such reduced species can have a LS GS due to the antiferromagnetic (AFM) interaction which can arise between opposite spins localized separately in metal and ligand orbitals. When this AFM stabilization outweighs the potential exchange stabilization if all unpaired spins were oriented in the same direction, the GS will be LS with a wavefunction requiring more than one determinant. ${ }^{47,116,119,120,122}$

From the perspective of SSB, we first investigate a set of metal complexes that was the focus of a recent work by Batista et al., in which redox potentials in non-aqueous 
TABLE VIII: $\left\langle S^{2}\right\rangle$ values calculated for the complexes in Ref. 12 . The $\kappa$-UOOMP2 value is in bold, as it is the only case in which SSB persists beyond the UHF level of theory.

\begin{tabular}{lllll}
\hline \hline & $S_{\text {exact }}^{2}$ & $S_{U H F}^{2}$ & $S_{U \text { B3LYP }}^{2}$ & $S_{\kappa U O O M P 2}^{2}$ \\
\hline $\mathrm{FeCp}_{2}$ & 0 & 1.21 & 0 & 0 \\
$\mathrm{FeCp}_{2}^{+}$ & 0.75 & 1.37 & 0.78 & 0.76 \\
$\mathrm{CoCp}_{2}$ & 0.75 & 1.54 & 0.77 & 0.76 \\
$\mathrm{CoCp}_{2}^{+}$ & 0 & 1.32 & 0 & 0 \\
$\mathrm{NiCp}_{2}$ & 2 & 2.01 & 2.01 & 2.01 \\
$\mathrm{NiCp}_{2}^{+}$ & 0.75 & 1.65 & 0.77 & 0.76 \\
\hline $\mathrm{FeCp}_{2}$ & 0 & 1.20 & 0 & 0 \\
$\mathrm{FeCp}_{2}{ }_{2}^{+}$ & 0.75 & 1.47 & 0.79 & 0.77 \\
$\mathrm{CoCp}_{2}{ }_{2}$ & 0.75 & 1.53 & 0.78 & 0.76 \\
$\mathrm{CoCp}_{2}^{*+}$ & 0 & 1.36 & 0 & 0.00 \\
$\mathrm{NiCp}{ }_{2}$ & 2 & 2.01 & 2.01 & 2.01 \\
$\mathrm{NiCp}{ }_{2}^{+}$ & 0.75 & 1.67 & 0.77 & 0.75 \\
\hline $\mathrm{Fe}(\mathrm{bpy})_{3}^{2+}$ & 0 & 3.71 & 0 & 0 \\
$\mathrm{Fe}(\mathrm{bpy})_{3}^{3+}$ & 0.75 & 3.43 & 0.77 & 0.79 \\
$\mathrm{Co}(\mathrm{bpy})_{3}^{2+}$ & 0.75 & 4.42 & 0.76 & 0.80 \\
$\mathrm{Co}(\mathrm{bpy})_{3}^{3+}$ & 0 & 2.66 & 0 & 0 \\
\hline \hline
\end{tabular}

solution were computed and compared to experimental measurements. ${ }^{12}$ This set included $\mathrm{MCp}_{2}$ and $\mathrm{MCp}_{2}^{*}$ where $\mathrm{M}=\mathrm{Fe}, \mathrm{Co}, \mathrm{Ni}$; and $\mathrm{M}^{\prime} \mathrm{bpy}_{3}$ where $\mathrm{M}^{\prime}=\mathrm{Fe}$, Co. $\mathrm{Cp}, \mathrm{Cp}^{*}$, bpy denote cyclopentadienyl, pentamethylCp, and bipyridine ligands, respectively. ${ }^{123}$ As shown in Table VIII, SSB in the UHF wavefunction occurs in nearly all species (with the exception of $\mathrm{NiCp}_{2}^{*}$ ). For the $\mathrm{Cp}$ and $\mathrm{Cp}^{*}$ complexes, UB3LYP and $\kappa$-UOOMP2 restore spin-purity in all cases. The implied SR character can be expected given the well-known stability of complexes like ferrocene.

For the tri-bpy complexes, the notably large UHF SSB is approximately restored in all cases with UB3LYP and $\kappa$-UOOMP2. Looking more closely at $\mathrm{Co}(\mathrm{bpy})_{3}^{2+}$, for which $\left\langle S^{2}\right\rangle_{\kappa U O O M P 2}$ calculated from the Slater determinant of optimized orbitals deviates by $6.6 \%$ from the exact value, we find that this deviation increases to $10.4 \%$ when evaluated with respect to the first-order wavefunction associated with $\kappa$-UOOMP2 (i.e. using Equation 6). To investigate the possibility that DE in the B3LYP functional has led to a bias toward spinsymmetry restoration for this $\mathrm{Co}(\mathrm{II})$ species, as seen previously for the stretched hydrogen-fluoride molecule in Fig. 3, we find $\left\langle S^{2}\right\rangle_{C A M-B 3 L Y P}=0.83$, identical to the value from the $\kappa$-UOOMP2 wavefunction. Fig. 7 also shows that SSB can be modulated by the amount of EXX included in global hybrid functionals, revealing that PBE with 20\% EXX yields SSB comparable to CAM-B3LYP, which appears sensible given that CAM-B3LYP has 19\% short-range EXX (and 65\% long-range EXX). Increasing \%EXX still further increases $\left\langle S^{2}\right\rangle$ towards the HF value. Recall that this arbitrariness is not unique to hybrid DFT

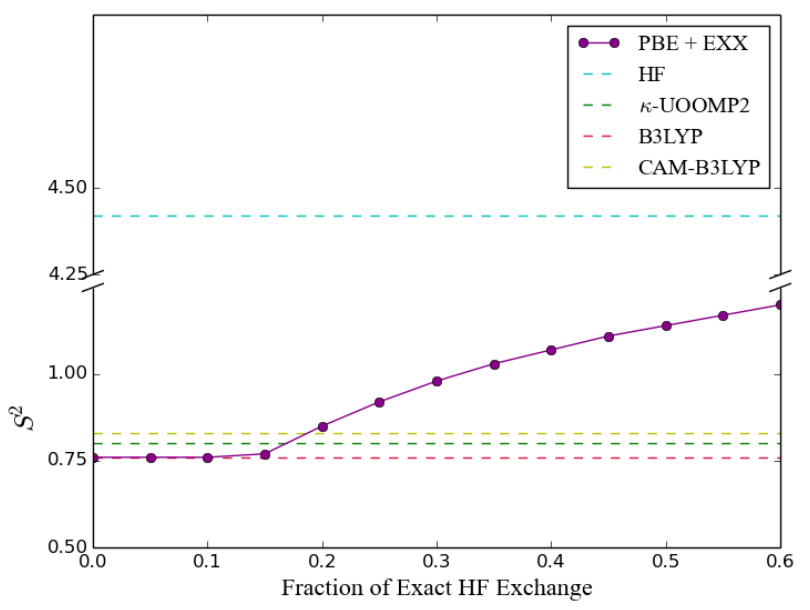

FIG. 7: $\left\langle S^{2}\right\rangle$ for the PBE-based hybrid functional as a function of exact $\mathrm{HF}$ exchange (EXX) fraction for $\mathrm{Co}(\mathrm{bpy})_{3}^{2+}$, compared with values from UHF, $\kappa$-UOOMP2, UB3LYP, and UCAM-B3LYP.

functionals, as the tendency toward SSB in $\kappa$-UOOMP2 is dependent on the value of the $\kappa$ regularizer: scanning $\kappa$ gives a full view of the symmetry-breaking landscape. ${ }^{52}$

Admittedly, it is difficult to draw conclusions regarding the $\mathrm{MR}$ character of the $\mathrm{Co}(\mathrm{bpy})_{3}^{2+}$ species. On the one hand, X-ray absorption spectra have been wellreproduced by linear combinations of simulated spectra from the LS (doublet) and HS (quartet) states, leading to the claim that $\mathrm{Co}(\mathrm{bpy})_{3}^{2+}$ is $57 \% \mathrm{HS}$ and $43 \%$ LS, whereas Co(bpy $)_{3}^{3+}$ is relatively more monoconfigurational ( $\sim 80 \% \mathrm{LS}) .{ }^{124}$ Taking these distributions and assuming the spectra were taken at room temperature implies spin gaps of roughly 0.01 and $0.03 \mathrm{eV}$ for the Co(II) and Co(III) species, respectively, which indeed would imply SC. On the other hand, that the bpy ligand is between $\mathrm{NH}_{3}$ and $\mathrm{CO}$ on the spectrochemical series suggests that the $10 \mathrm{Dq}$ should be relatively large due to $\pi$-backbonding, and therefore SSB is likely to be restored with a suitable level of dynamical correlation (and indeed it is with B3LYP). It is also true that UB3LYP was used in Ref. 12 and the calculated $\mathrm{Co}(\mathrm{III}) \rightarrow \mathrm{Co}(\mathrm{II})$ reduction potential matched the experimental value to a very high degree of accuracy (within a tenth of an $\mathrm{eV}$, when triple- $\zeta$ basis sets were used). Thus, negligible SSB from B3LYP, and worst case deviations of around $10 \%$ from CAM-B3LYP and the $\kappa$-UOOMP1 wavefunction, suggest that this complex is predominately of SR character, with redox-innocent bpy ligands.

We now turn to Fe complexes with terpyridine (tpy) or porphyrin ligands, which due to the more delocalized ligand frameworks are expected to have a relatively lowerlying $\pi^{*}$ orbital than bpy. Both complexes are efficient electrocatalysts for the $\mathrm{CO}_{2}$ reduction reaction $(\mathrm{CO} 2 \mathrm{RR})$ to $\mathrm{CO}$. An important feature of $\mathrm{CO} 2 \mathrm{RR}$ electrocatalysts is the substrate selectivity of $\mathrm{CO}_{2}$ against the hydrogen evolution reaction (HER) because proton-coupled $\mathrm{CO}_{2}$ 

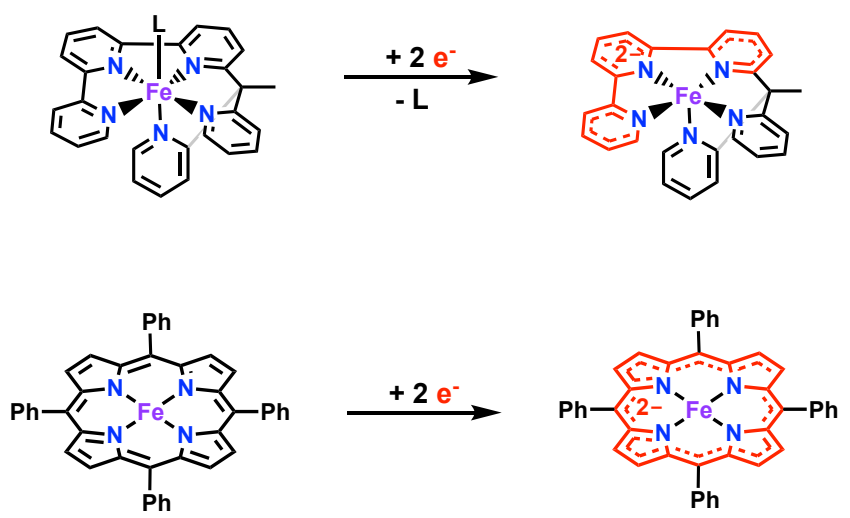

FIG. 8: Schematic view of the reduction process of Fe tpy and porphyrin complexes, as part of their CO2RR catalytic cycles. The red moiety indicates the location of the excess electrons, and $\mathrm{L}=\mathrm{CH}_{3} \mathrm{CN}$.

reduction and direct proton reduction occur at similar potentials. The incorporation of a redox-active ligand, which when reduced yields a relatively Lewis-acidic metal center, results in the metal favoring $\mathrm{CO}_{2}$ binding over protonation (formation of a metal-hydride is the first step in the HER mechanism). This origin of productselectivity has been established for the $\operatorname{Re}($ bpy $)(\mathrm{CO})_{3} \mathrm{Cl}$ complex $^{117,125}$ and also for iron polypyridine ${ }^{119,120,126}$ and porphyrin ${ }^{116}$ catalysts. Furthermore, placement of the extra electron in a $\pi^{*}$ orbital that is lower-lying than a virtual $d$ orbital results in milder reduction potentials - another desirable feature of an electrocatalyst - that are made milder still due to the additional stabilization from the M-L AFM coupling.

Combined spectroscopic and computational work conducted by Neese and coworkers ${ }^{116}$ suggests that the active species of the popular FeTPP catalyst (TPP = tetraphenylporphyrin) is an intermediate-spin $\mathrm{Fe}(\mathrm{II})$ center that is antiferromagnetically coupled to a porphyrin diradical anion. The FeTPP catalyst and its derivatives are among the most active $\mathrm{CO}_{2}$ reduction catalysts. ${ }^{112,115,127}$ Recent work by Derrick et al. shows that a tpy-based ligand framework (tpyPY2Me) in combination with an iron center is an efficient $\mathrm{CO}_{2}$ reduction catalyst at a low overpotential. ${ }^{120}$ The doubly reduced active form of the catalyst is a singlet with a doublyreduced tpy ligand strongly coupled to an intermediate spin Fe(II) center. This electronic structure was established based on both computational and spectroscopical evidence. Both complexes and their reduction reactions are depicted in Figure 8.

Table IX shows the SSB behavior for the Fe(II)-tpy species with net charge, $\mathrm{n}=2+, 1+$, and 0 . The first reduction is known to occupy the non-innocent tpy $\pi^{*}$ orbital, leaving the $\mathrm{d}^{6} \mathrm{Fe}(\mathrm{II})$ center closed-shell, and thus forming an overall doublet. Consistent with this picture, both the $n=2+$ and singly-reduced complexes, while severely spin-contaminated at the UHF level, do not exhibit significant SSB when accounting for dynamic cor- relation via B3LYP, CAM-B3LYP, and B5050LYP functionals. In contrast, the second reduction is accompanied by loss of a ligand and a spin transition of the iron center from LS to intermediate spin (i.e. $\mathrm{S}_{\mathrm{Fe}}=1$ ), while the twice-reduced tpy $\left(S_{\text {tpy }}=1\right)$ couples to the metal center to form an overall singlet. This AFM coupling of opposite spins separately localized on metal and ligand requires a MR description (for fundamentally the same reason that open-shell singlet biradicals require two determinants), and manifests as SSB which persists upon inclusion of dynamic correlation with the three DFT functionals investigated. As expected, the calculated $\left\langle S^{2}\right\rangle$ value increases with \%EXX in the hybrid functional.

In the case of the iron porphyrin, here modelled without the phenyl groups (denoted FeP), the neutral complex has a triplet GS. ${ }^{128}$ As indicated by the complete restoration of the UHF SSB with all DFT functionals and $\kappa$-UOOMP2, shown in Table $\mathrm{X}$, this species is predicted to have a SR electronic structure. Both the first and second reductions are ligand-centered and result in M-L AFM coupling which has been observed experimentally. The SSB which persists from HF through all DFT methods (increasing, as in the Fe tpy systems above, with \% EXX), for both $\mathrm{FeP}^{-}$and $\mathrm{FeP}^{2-}$ corroborate the presence of M-L AFM coupling involving the $S_{F e}=1$ center and the reduced, non-innocent porphyrin.

As first encountered in Ref. 54, $\kappa$-UOOMP2 predicts unphysical GSs for the FeP species; therein, the DIIS solver was used along with an appropriate initial orbital guess to converge on the correct state. Indeed, for the twice-reduced Fe tpy and FeP species studied presently, use of the GDM solver yielded spin-pure GSs inconsistent with the AFM-coupled states deduced from experimental measurements and predicted from B3LYP, CAMB3LYP, and B5050LYP functionals. A detailed study on this topic is forthcoming, so at present we simply report that use of the DIIS solver enabled us to obtain the expected redox states and associated SSB behavior from $\kappa$-UOOMP2 (though these states are higher in energy than those obtained from the GDM solver).

Finally we remark that MR character arises also when catalysts such as metalloporphyrins bind small-molecule substrates such as $\mathrm{O}_{2}$ and $\mathrm{NO}^{129-132}$. These are of great biological relevance, and deserve further investigation alongside catalytic systems for CO2RR and other desirable reactions. The present results show that SSB from these hybrid DFT functionals is a computationally inexpensive hallmark which can be used in future in silico catalyst design projects, and can inform, if not replace, chemical intuition regarding the possible non-innocence of novel ligand frameworks.

\section{AFM coupling in metal-metal dimers}

The quintessential example of AFM coupling are multimetal compounds. ${ }^{133-135}$ As a simple example system we consider the four smallest $\mathrm{Mn}(\mathrm{III})-\mathrm{Mn}(\mathrm{IV})$ compounds 
TABLE IX: $\left\langle S^{2}\right\rangle$ values of dication, singly- and doubly-reduced Fe-tpy complexes. $\omega$ B97X-D geometries are used, taken from Ref. 120.

\begin{tabular}{lllllll}
\hline \hline$\left[\mathrm{Fe}(\mathrm{II})\left(\mathrm{tpyPY} 2 \mathrm{Me}^{2-}\right)\right]^{n}$ & $S_{\text {exact }}^{2}$ & $S_{U H F}^{2}$ & $S_{U B 3 L Y P}^{2}$ & $S_{U C A M-B 3 L Y P}^{2}$ & $S_{U B 5050 L Y P}^{2}$ & $S_{\kappa U O O M P 2}^{2}$ \\
\hline $\mathrm{n}=2+$ & 0.00 & 2.85 & 0.00 & 0.00 & 0.00 & 0 \\
$\mathrm{n}=1+$ & 0.75 & 2.94 & 0.76 & 0.77 & 0.78 & $0.78^{\dagger}, 0.86^{*}$ \\
$\mathrm{n}=0$ & 0.00 & 4.03 & 1.48 & 1.76 & 1.94 & $1.94^{\dagger}, 0.00^{*}$ \\
\hline \hline${ }^{\dagger}$ DIIS solver for $\kappa$-UOOMP2 & & & & & \\
& ${ }^{*}$ GDM solver for $\kappa$-UOOMP2 & & & & &
\end{tabular}

TABLE X: $\left\langle S^{2}\right\rangle$ values of neutral, singly- and doubly-reduced iron porphyrin $(\mathrm{FeP})$ complexes. LRC- $\omega$ PBEh/def2-SV(P) geometries are used.

\begin{tabular}{|c|c|c|c|c|c|c|}
\hline$[\mathrm{FeP}]^{n}$ & $S_{\text {exact }}^{2}$ & $S_{U H F}^{2}$ & $S_{U B 3 L Y P}^{2}$ & $S_{U C A M-B 3 L Y P}^{2}$ & $S_{U B 5050 L Y P}^{2}$ & $S_{\kappa U O O M P 2}^{2}$ \\
\hline $\mathrm{n}=0$ & 2 & 3.98 & 2.01 & 2.01 & 2.01 & $2.01^{\dagger}, 2.07^{*}$ \\
\hline $\mathrm{n}=1-$ & 0.75 & 3.24 & 1.61 & 1.69 & 1.74 & $1.88^{\dagger}, 0.87^{*}$ \\
\hline $\mathrm{n}=2-$ & 0 & 3.03 & 1.76 & 2.00 & 2.26 & $1.93^{\dagger}, 0.00^{*}$ \\
\hline
\end{tabular}

from Ref. 136, which each have two bridging oxygens directly connecting the $\mathrm{Mn}$ centers. The $\left\langle S^{2}\right\rangle_{U B 3 L Y P}$ values for compounds numbered $5,6,7$, and 11 are all 3.8 , which is far from the exact value of 0.75 for these experimentally-assigned doublet species. We confirmed that the spin-densities on the Mn atoms are 4 and -3 in all cases (we note that a HS, octet calculation needed to be performed first, and the resulting density was used to initialize a subsequent calculation of the doublet state), indicating strong AFM coupling. As expected, for these types of multi-metal states, MR methods with large active spaces are required to obtain exchange-coupling parameters that agree with experiment ${ }^{40}$. The coupling constant and relative spin-state orderings are very sensitive to the DFT functional employed, ${ }^{137}$ implying that caution and careful, system-specific benchmarking is required if such methods are to be used.

\section{F. A cautionary coda on the use of MP2 and double-hybrid functionals on single-reference organometallics}

In this section we emphasize that even in the absence of persistent SSB (i.e. for species well-described by a single determinant), computational methods should be chosen with care. In other words, in our view categorizing a system as either MR or not is only the first step to quantitatively accurate predictions. As an illustrative example, we focus on metal complexes with strong-field CO ligands, which are of primary importance in organometallic chemistry. As discussed above, the MO diagrams of these complexes are characterized by large 10Dq values which result in spin-pure, LS GSs. However, Figure 9 shows that double hybrid density functionals (DHDFs) which incorporate MP2 correlation energies consistently overestimate experimental ligand dissociation energies ${ }^{35,138}$

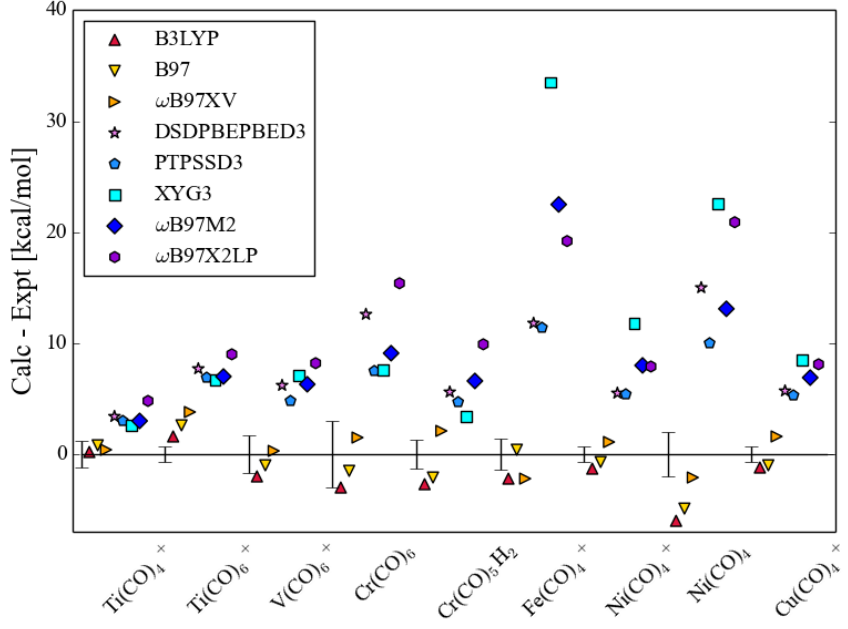

FIG. 9: Ligand-dissociation energies, comparison of calculated values extrapolated to the CBS limit vs experimental. Geometries optimized at the UB3LYP-DKH/cc-pVTZ-DKH level; single-points with the indicated functional in the def2-QZVPP basis without scalar relativistic effects. These relativistic effects are very small for $3 d$ metal carbonyls ${ }^{141}$, and the present calculations without scalar relativity agree quite well with DKH calculations (albeit in a triple- $\zeta$ basis) from Ref. 34 .

for M-CO complexes. While one might expect that the highest rung of Jacob's ladder ${ }^{139,140}$ would provide the most accurate results, for these organometallic compounds DHDFs drastically underperform simpler functional forms, e.g. the global hybrids B3LYP and B97, and the RSH $\omega \mathrm{B} 97 \mathrm{X}-\mathrm{V}$.

Next, we consider the 3d metal complexes from Ref. 34 with gas-phase experimental ligand-dissociation measurements, and select only those for which DLPNOCCDS(T)/CBS yields accurate results. The six suit- 


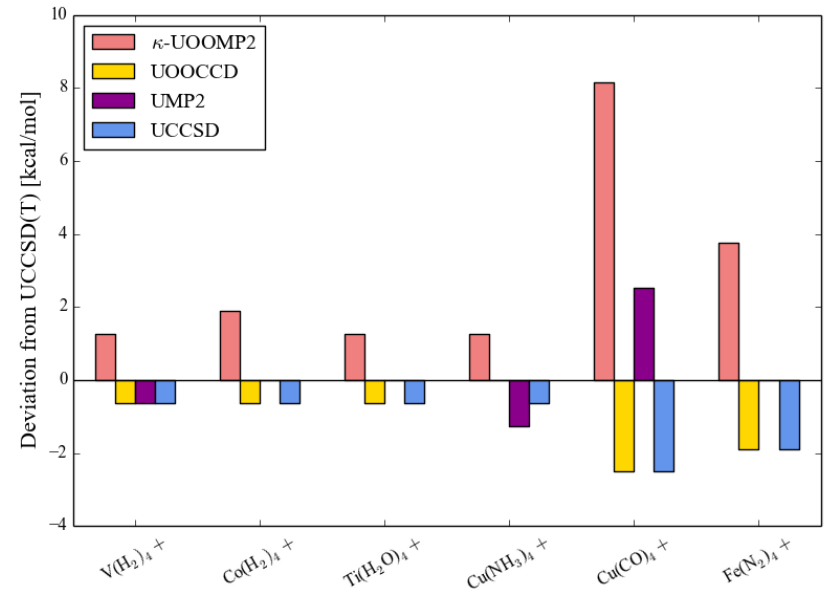

FIG. 10: Deviations of the ligand-dissociation energies $[\mathrm{kcal} / \mathrm{mol}]$ calculated with various methods from $\operatorname{UCCSD}(T)$, in the def2-SV(P) basis. A negative deviation denotes underbinding.

able compounds are $\mathrm{V}\left(\mathrm{H}_{2}\right)_{4}^{+}, \mathrm{Co}\left(\mathrm{H}_{2}\right)_{4}^{+}, \quad \mathrm{Ti}\left(\mathrm{H}_{2} \mathrm{O}\right)_{4}^{+}$, $\mathrm{Cu}\left(\mathrm{NH}_{3}\right)_{4}^{+}, \mathrm{Cu}(\mathrm{CO})_{4}^{+}$, and $\mathrm{Fe}\left(\mathrm{N}_{2}\right)_{4}^{+} \cdot \mathrm{H}_{2} \mathrm{O}$ is a $\pi$-donor and $\mathrm{NH}_{3}$ ligands can only engage in $\sigma$-bonding with the metal. $\mathrm{H}_{2}, \mathrm{~N}_{2}$, and $\mathrm{CO}$ ligands are $\pi$-acceptors, in order of increasing back-bonding strength (see Table S2). Fig. 10 compares, in the def2-SV(P) basis, the deviations of the ligand-dissociation energies from UMP2, $\kappa$-UOOMP2, UOOCCD, and UCCSD calculations with $\operatorname{UCCSD}(\mathrm{T})$. Evidently, while reasonable accuracy for transition metal chemistry $\left(\sim 2-3 \mathrm{kcal} / \mathrm{mol}^{37}\right)$ is obtained for the complexes with ligands that can only $\sigma$-donate or weakly $\pi$-accept, $\mathrm{Cu}(\mathrm{CO})_{4}^{+}$yields the largest error with MP2 methods (interestingly orbital optimization makes the accuracy worse). MP2, like the DHDFs using its correlation energy, overestimates the liganddissociation energy vs experiment. Neese et al. also found that MP2 and OOMP2 produce large (4.8 - 60 $\mathrm{kcal} / \mathrm{mol}$ ) errors vs $\operatorname{CCSD}(\mathrm{T})$ for CO dissociation of four $\mathrm{Cr}$ and $\mathrm{Ni}$ complexes; ${ }^{142}$ similarly, Hyla-Kryspin and Grimme found for MP2-calculated CO-dissociation enthalpies an average overestimation of $19.3 \mathrm{kcal} / \mathrm{mol}$ over a set of $73 \mathrm{~d}$ metal carbonyl species (interestingly, while MP3 led to underestimations of even larger average magnitude, the performance of spin-component-scaled MP2 and MP3 was more promising, with MAEs of 9.6 and 3.7 $\mathrm{kcal} / \mathrm{mol}$, respectively). ${ }^{143}$

We hypothesize that while the pair-wise additive approximation for the correlation energy of MP2 theory can adequately describe $\sigma$-bond only ligands (in the absence of static correlation), it cannot be expected to produce quantitative results for 4- and 6-electron-like interactions involving both $\sigma$-donation and $\pi$-backbonding, regardless of the orbital set employed. $\sigma$-donation involves one electron pair, while $\pi$-backbonding can additionally involve either one or two more electron pairs, depending on the number of available donor-acceptor orbitals. In the case of a metal-carbonyl bond, $\pi$-backbonding can involve two pairs of electrons backdonated into the degenerate $\pi_{x}^{*}$ and $\pi_{y}^{*}$ orbitals of $\mathrm{CO}$, resulting in a bond that effectively involves 6 correlated electrons in total.

This reasoning begs the following question: For metal carbonyl complexes, or more generally for metal complexes with strong-field ligands which can significantly $\pi$ accept (including polypyridines and porphyrins relevant in previous sections of this work), will the SSB behavior of $\kappa$-UOOMP2 be compromised due to its inability to describe the inter-pair correlations involved in dative bonds characterized by simultaneous $\sigma$-donation and $\pi$ backdonation? If so, how can SSB due to this be distinguished from SSB due to genuine MR character? The first point is that these inter-pair effects are, in general, smaller than single pair correlation energies. Moreover, when inter-pair correlations are physically significant (as we argue to be the case in organometallic compounds), the gap between LS and HS states is typically already large, as in the $\mathrm{Fe}(\mathrm{II}) \mathrm{CO}_{6}^{2+}$ systems discussed earlier, rendering spin-state mixing an irrelevant concern in most situations. However, this may not be the case for complexes with redox-noninnocent ligands, e.g. Fe tpy and porphyrin, for which SSB from DFT orbitals is recommended. Work is ongoing to disentangle errors due to $\kappa$-regularization and the MP2 ansatz itself.

\section{CONCLUSIONS}

While transition metal chemistry has a reputation for being challenging for computational quantum chemistry models, one has to look hard for SC in the GSs of monometal transition metal complexes. This is because for small $10 \mathrm{Dq}$ values the HS state (which is, in general, SR) is favored due to exchange stabilization of same-spin electrons and the energy "saving" from not having to pair electrons; for large 10Dq values the LS is energetically far-below the HS state which prevents the latter from contaminating the total spin of the former, leading again to a SR state. The latter situation occurs when $4 \mathrm{~d}$ and $5 \mathrm{~d}$ transition metals are involved. One of the goals of this work, though, is to find relevant situations in which static correlation is present in molecules. We have encountered MR character in:

1. metal-hydride diatomics courtesy of the inapplicability of the Jahn-Teller theorem to linear systems, in which spatial symmetry mandates two important determinants for both $\mathrm{TiH}$ and $\mathrm{CoH}$. For less electronegative $3 \mathrm{~d}$ metals, i.e. going from $\mathrm{Ni}$ to Co to, mostly strikingly, Fe, we find increasingly non-negligible LUNOs due to the narrowing gap between the non-bonding metal 4s-3d shell and the antibonding orbital (involving metal $\mathrm{d}_{z}^{2}$ and hydrogen 1s) which opens the door to SSB due to the intrusion of higher-spin-state character.

2. metal complexes with higher coordination number and low (i.e. $\mathrm{Fe}(\mathrm{I})$ or $\mathrm{Mn}(\mathrm{I})$ ) oxidation states, 
which exhibit very weak bond energies (tens of $\mathrm{kJ} / \mathrm{mol}$ ) due to weak-field ( $\sigma$-donation only) ligands and metals with singly-occupied $4 \mathrm{~s}$ orbital preventing favorable dative bonding. While less likely to exist in solution, such weak bonding can be encountered in the gas-phase (in, e.g., metalorganic frameworks or atmospheric chemistry).

3. molecules which exhibit AFM coupling resulting in LS GSs. We have demonstrated that this can occur in reduced states of homogeneous catalysts containing redox-noninnocent ligands such as terpy and porphyrin, and also in oxygen-bridged bimetallic $\mathrm{Mn}(\mathrm{III})-\mathrm{Mn}(\mathrm{IV})$ dimers.

For para-benzyne along with stretched $\mathrm{H}_{2}$ and $\mathrm{HF}$, the SSB behavior and NOON predictions from DFT orbitals agree quite well with those of ASCI (a near-exact FCI approximation). As expected, increasing the fraction of EXX in hybrid functionals pushed CF points to shorter bond lengths, and increased the amount of artificial SSB (i.e. LUNOs in excess of the exact value) approaching that of UHF (i.e. 100\% EXX). It is remarkable that computing $\left\langle S^{2}\right\rangle$ from the KS orbitals (which represent the solution of a non-interacting problem) yields results similar to those obtained from approximate (e.g. $\kappa$-UOOMP2) wavefunction methods, and NOONs in close agreement with those from ASCI. However, as the case of stretched HF reveals, when pure and common hybrid DFT orbitals are used to compute $\left\langle S^{2}\right\rangle$ and NOONs, the static correlation error is intertwined with $\mathrm{DE}$ which leads to erroneous results. In such cases, $\kappa$-UOOMP2, which is free of $\mathrm{DE}$, is formally more reliable (in addition to the fact that $\left\langle S^{2}\right\rangle$ and NOONs are well-defined). We note that rangeseparated hybrids and functionals with a high percentage of EXX are lower-scaling alternatives in which DE is much-reduced, and the use of orbitals from optimallytuned RSH functionals may be a potentially interesting way forward in certain cases.

Regarding the diagnosis of static correlation, SSB at the UB3LYP and $\kappa$-UOOMP2 levels is shown to be insufficient in case 1 from above, as it does not account for a wavefunction that must be multi-configurational to respect spatial symmetry, and generally in LS states which are not the GS due to variational collapse. In these special cases, SSB should be used in conjunction with NOONs from a MR method, ideally one with an appropriate treatment of dynamic correlation. Methods that use a HS reference and apply spin-flip excitations to generate the Hilbert space of lower spin multiplicities appear to be good candidates to, e.g., probe the possible multi-configurational nature of a LS excited state, and have the advantage of a black-box selection of the active space orbitals involved in spin-unpairing from the target LS state.

Another important takeaway suggested by this work is that SC is a term that demands clarification, especially in the context of transition metals. The term is frequently used as a synonym of static correlation and
MR character, and we have presented SSB as an intuitive and meaningful diagnostic for the GS when spatial symmetry is not an issue. Having established a connection with chemically-revealing models such as ligand-field and molecular orbital theories, a picture is painted which suggests that, fundamentally, static correlation in transition metal systems involves the same phenomenon as in organic molecules, e.g. biradicaloids. ${ }^{52,144}$

In contrast, evidently it is the dynamic correlation which presents additional difficulties compared to the case of typical organic molecules. For organometallics, 4- and 6-electron-like interactions (or at least inter- electron pair correlations) as relevant to $\pi$-donation or $\pi$ backdonation on top of $\sigma$-donation appear to be important, as illustrated by the failure of MP2 and MP2based DHDFs in predicting experimental ligand dissociation energies for metal carbonyl complexes. A second idea which supports this conclusion, while admittedly less concrete, is that large errors in DLPNO-CCSD $(\mathrm{T})$ calculations were found when the UHF solution exhibited significant spin-contamination. It is our expectation that when orbitals obtained from theories which include (even approximately) dynamical correlation such as DFT and $\kappa$-UOOMP2 are employed, subsequent CC predictions will improve in accuracy, as has already been seen in main group molecules. ${ }^{145,146}$ Thus, in our view, the common notion that transition metals are difficult for traditional electronic structure methods is due to both static and dynamic correlation, yet we stress our finding that truly MR situations are encountered only in the special cases enumerated above (and perhaps a few others), making the proper treatment of dynamic correlation more important in most commonly-encountered cases.

There is also an important connection between a theoretical method's ability to capture dynamical correlation and the ability of SSB to imply MR character, which depends on the accurate prediction of relative energies between two (or possibly more) spin states. In the limit of no correlation other than the exchange interaction between same-spin electrons (required by Fermi statistics/Pauli-exclusion), as is the case in HF theory, HS states are artificially favored relative to LS states. For a system with a LS GS (determined experimentally or by an exact theoretical method), HF (and often CASSCF with small active spaces) will significantly underestimate the LS-HS gap or even incorrectly predict a HS GS. In such cases, the SSB implied by the HF LS wavefunction will be artificially large due to the unphysical inclusion of or collapse to the HS state. The idea behind using orbitals optimized from Kohn-Sham DFT or $\kappa$-UOOMP2 is essentially an attempt to more accurately describe relative spin-state energetics via the (approximate) inclusion of dynamic correlation, which enables true MR character to be reliably diagnosed.

And yet the difficulty involved in accurately predicting LS-HS gaps (in a reasonable amount of time) cannot be overestimated. With regard to DFT, the issue stems from the systematic overstabilization of HS states as the 
fraction of EXX is increased. Pure DFT functionals, e.g. PBE, BLYP, or BP86, are justifiably advantageous when investigating transition metal systems in the sense that the resulting orbitals are likely to be free of "artificial" SSB; subsequent PT or CC will converge more quickly, and there is no arbitrariness in, e.g., how much EXX to include. However, pure functionals are plagued by high levels of DE, and as a result are known to yield unphysical charge and spin densities, underestimate barrier heights, etc. With regard to $\kappa$-UOOMP2, we recall i) the large 3 $\mathrm{eV}$ error in the vertical spin gap of the iron hexacarbonyl species (vs CCSD(T) in the same basis), and ii) the spurious closed-shell GSs implied for the twice-reduced Fe tpy and porphyrin species, which are known experimentally to be open-shell singlet states with AFM coupling between metal and ligand. Both i) and ii) involve the over-stabilization of closed-shell LS states in complexes with strong-field ligands which form bonds to metal ions involving simultaneous $\sigma$-donation and $\pi$-backdonation. Evidently, for these types of organometallic complexes, the fact that dynamic correlations among multiple electron pairs are entirely missing in MP2 approaches (and thus to some extent in DHDFs incorporating MP2 correlation) has a detrimental impact on the accuracy of predictions regarding thermochemistry (Figure 9), relative spin-state energetics, and the presence of static correlation via SSB.

Further development and assessment of SR methods, in addition to local correlation functionals, capable of describing this type of dynamic correlation relevant to transition metal bonding is clearly necessary. CCSD and $\operatorname{CCSD}(\mathrm{T})$ are limited by their high scaling. The direct variant of the Random Phase Approximation (dRPA), ${ }^{147}$ which can be implemented to scale as the fourth power of system size, ${ }^{148}$ has been shown to be equivalent to ring-CCD ${ }^{149}$ and therefore includes terms approximately correlating excitations involving more than one electron pair. dRPA has shown promising accuracy for bond energies of metal carbonyl complexes (among others). ${ }^{150}$ On the other hand, the improved description of the dissociation limit within the RPA formalism comes at the expense of DE. ${ }^{151}$ OORPA approaches have been developed, ${ }^{152}$ which do not seem to require regularization. UOOCCD and MP3 also approximately include inter-pair excitations, and the scaling of these methods might be reduced by localized approximations or tensor decompositions of the two-electron integrals, enabling their use in DHDFs. Our data suggests, though it remains to be seen, that the performance of DHDFs incorporating such correlation energies (as in, e.g., Ref. 153) instead of MP2 may yield improved accuracy for organometallic thermochemistry.

\section{ACKNOWLEDGMENTS}

JS thanks Romit Chakraborty for insightful discussions. This work was supported by the Director, Office of Science, Office of Basic Energy Sciences, of the U.S. Department of Energy under Contract No. DE-AC02$05 \mathrm{CH} 11231$.

\section{DATA AVAILABILITY}

The data that supports the findings of this study are available within the article [and its supplementary material].

${ }^{1}$ C. J. Cramer and D. G. Truhlar, "Density functional theory for transition metals and transition metal chemistry," Phys. Chem. Chem. Phys. 11, 10757-10816 (2009).

${ }^{2}$ K. D. Vogiatzis, M. V. Polynski, J. K. Kirkland, J. Townsend, A. Hashemi, C. Liu, and E. A. Pidko, "Computational approach to molecular catalysis by $3 \mathrm{~d}$ transition metals: Challenges and opportunities," Chem. Rev. 119, 2453-2523 (2018).

${ }^{3}$ F. Furche and J. P. Perdew, "The performance of semilocal and hybrid density functionals in $3 \mathrm{~d}$ transition-metal chemistry," $\mathrm{J}$. Chem. Phys. 124, 044103 (2006).

${ }^{4}$ Y. A. Aoto, A. P. de Lima Batista, A. Köhn, and A. G. de Oliveira-Filho, "How to arrive at accurate benchmark values for transition metal compounds: computation or experiment?" J. Chem. Theory Comput. 13, 5291-5316 (2017).

${ }^{5}$ J. Shee, B. Rudshteyn, E. J. Arthur, S. Zhang, D. R. Reichman, and R. A. Friesner, "On achieving high accuracy in quantum chemical calculations of $3 \mathrm{~d}$ transition metal-containing systems: A comparison of auxiliary-field quantum monte carlo with coupled cluster, density functional theory, and experiment for diatomic molecules," J. Chem. Theory Comput. 15, 2346-2358 (2019).

${ }^{6}$ S. J. Bennie, D. Collison, and J. J. McDouall, "Electronic and magnetic properties of kremer's tris-hydroxo bridged chromium dimer: A challenge for dft," J. Chem. Theory Comput. 8, 49154921 (2012).

${ }^{7}$ P. E. Siegbahn, "The performance of hybrid dft for mechanisms involving transition metal complexes in enzymes," J. Biol. Inorg. Chem. 11, 695-701 (2006).

${ }^{8}$ M. Lundberg and P. E. Siegbahn, "Agreement between experiment and hybrid dft calculations for o-h bond dissociation enthalpies in manganese complexes," J. Comput. Chem. 26, 661667 (2005).

${ }^{9}$ P. E. Siegbahn, "An energetic comparison of different models for the oxygen evolving complex of photosystem ii," J. Am. Chem. Soc. 131, 18238-18239 (2009).

${ }^{10} \mathrm{M}$. Reiher, "Relativistic douglas-kroll-hess theory," Wiley Interdiscip. Rev. Comput. Mol. Sci. 2, 139-149 (2012).

${ }^{11} \mathrm{~F}$. Neese, "Efficient and accurate approximations to the molecular spin-orbit coupling operator and their use in molecular gtensor calculations," J. Chem. Phys. 122, 034107 (2005).

${ }^{12}$ S. J. Konezny, M. D. Doherty, O. R. Luca, R. H. Crabtree, G. L. Soloveichik, and V. S. Batista, "Reduction of systematic uncertainty in dft redox potentials of transition-metal complexes," J. Phys. Chem. C 116, 6349-6356 (2012).

${ }^{13}$ F. Arrigoni, R. Breglia, L. D. Gioia, M. Bruschi, and P. Fantucci, "Redox potentials of small inorganic radicals and hexaaquo complexes of first-row transition metals in water: A dft study based on the grand canonical ensemble," J. Phys. Chem. A 123, 6948-6957 (2019).

${ }^{14}$ A. J. Cohen, P. Mori-Sánchez, and W. Yang, "Fractional spins and static correlation error in density functional theory," J. Chem. Phys. 129, 121104 (2008), https://doi.org/10.1063/1.2987202.

${ }^{15}$ A. J. Cohen, P. Mori-Sánchez, and W. Yang, "Insights into current limitations of density functional theory," Science 321, 792-794 (2008). 
${ }^{16}$ E. R. Davidson and W. T. Borden, "Symmetry breaking in polyatomic molecules: real and artifactual," J. Phys. Chem. 87, 4783-4790 (1983).

${ }^{17} \mathrm{X}$. Li and J. Paldus, "A unitary group based open-shell coupled cluster study of vibrational frequencies in ground and excited states of first row diatomics," J. Chem. Phys. 104, 9555-9562 (1996).

${ }^{18}$ H. Fukutome, "Unrestricted hartree-fock theory and its applications to molecules and chemical reactions," Int. J. Quantum Chem. 20, 955-1065 (1981).

${ }^{19} \mathrm{~J}$. Struber and J. Paldus, Fundamental World of Quantum Chemistry: A Tribute to the Memory of Per-Olov Löwdin Volume III, edited by E. J. Brändas and E. S. Kryachko, Vol. 1 (Kluwer Academic Publishers, Dordrecht, Netherlands, 2003) pp. 67-139.

${ }^{20} \mathrm{~J}$. F. Harrison, "Electronic structure of diatomic molecules composed of a first-row transition metal and main-group element (hf)," Chem. Rev. 100, 679-716 (2000).

${ }^{21} \mathrm{X}$. Xu, W. Zhang, M. Tang, and D. G. Truhlar, "Do practical standard coupled cluster calculations agree better than kohnsham calculations with currently available functionals when compared to the best available experimental data for dissociation energies of bonds to $3 \mathrm{~d}$ transition metals?" J. Chem. Theory Comput. 11, 2036-2052 (2015).

${ }^{22}$ E. R. Johnson and A. D. Becke, "Communication: Dft treatment of strong correlation in 3d transition-metal diatomics," J. Chem. Phys. 146, 211105 (2017).

${ }^{23}$ J. L. Bao, S. O. Odoh, L. Gagliardi, and D. G. Truhlar, "Predicting bond dissociation energies of transition-metal compounds by multiconfiguration pair-density functional theory and second-order perturbation theory based on correlated participating orbitals and separated pairs," J. Chem. Theory Comput. 13, 616-626 (2017).

${ }^{24}$ J. J. Determan, K. Poole, G. Scalmani, M. J. Frisch, B. G. Janesko, and A. K. Wilson, "Comparative study of nonhybrid density functional approximations for the prediction of $3 \mathrm{~d}$ transition metal thermochemistry," J. Chem. Theory Comput. 13, 4907-4913 (2017).

${ }^{25}$ Z. Fang, M. Vasiliu, K. A. Peterson, and D. A. Dixon, "Prediction of bond dissociation energies/heats of formation for diatomic transition metal compounds: Ccsd (t) works," J. Chem. Theory Comput. 13, 1057-1066 (2017)

${ }^{26}$ D. Hait, N. M. Tubman, D. S. Levine, K. B. Whaley, and M. Head-Gordon, "What levels of coupled cluster theory are appropriate for transition metal systems? a study using nearexact quantum chemical values for $3 \mathrm{~d}$ transition metal binary compounds," J. Chem. Theory Comput. 15, 5370-5385 (2019).

${ }^{27}$ K. T. Williams, Y. Yao, J. Li, L. Chen, H. Shi, M. Motta, C. Niu, U. Ray, S. Guo, R. J. Anderson, et al., "Direct comparison of many-body methods for realistic electronic hamiltonians," Phys. Rev. X 10, 011041 (2020).

${ }^{28}$ M. D. Morse, "Predissociation measurements of bond dissociation energies," Acc. Chem. Res. 52, 119-126 (2018).

${ }^{29}$ K. Raghavachari, G. W. Trucks, J. A. Pople, and M. HeadGordon, "A fifth-order perturbation comparison of electron correlation theories," Chem. Phys. Lett. 157, 479-483 (1989).

${ }^{30} \mathrm{M}$. Hanauer and A. Koehn, "Perturbative treatment of triple excitations in internally contracted multireference coupled cluster theory," J. Chem. Phys. 136, 204107 (2012).

${ }^{31}$ W. Jiang, N. J. DeYonker, and A. K. Wilson, "Multireference character for $3 \mathrm{~d}$ transition-metal-containing molecules," J. Chem. Theory Comput. 8, 460-468 (2012).

${ }^{32}$ B. M. Hockin, C. Li, N. Robertson, and E. Zysman-Colman, "Photoredox catalysts based on earth-abundant metal complexes," Catal. Sci. Technol. 9, 889-915 (2019).

${ }^{33} \mathrm{H}$. A. Jahn and E. Teller, "Stability of polyatomic molecules in degenerate electronic states-i - orbital degeneracy," Proc. Math. Phys. Eng. Sci. 161, 220-235 (1937).

${ }^{34}$ B. Rudshteyn, D. Coskun, J. L. Weber, E. J. Arthur, S. Zhang, D. R. Reichman, R. A. Friesner, and J. Shee, "Predicting ligand-dissociation energies of $3 \mathrm{~d}$ coordination complexes with auxiliary-field quantum monte carlo," J. Chem. Theory Comput. (2020).

${ }^{35}$ M. Rodgers and P. Armentrout, "Cationic noncovalent interactions: energetics and periodic trends," Chem. Rev. 116, 56425687 (2016).

${ }^{36}$ B. O. Roos, P. R. Taylor, and P. E. Sigbahn, "A complete active space scf method (casscf) using a density matrix formulated super-ci approach," Chem. Phys. 48, 157-173 (1980).

${ }^{37}$ N. J. DeYonker, K. A. Peterson, G. Steyl, A. K. Wilson, and T. R. Cundari, "Quantitative computational thermochemistry of transition metal species," J. Phys. Chem. A 111, 11269-11277 (2007).

${ }^{38}$ L. Freitag, S. Knecht, S. F. Keller, M. G. Delcey, F. Aquilante, T. B. Pedersen, R. Lindh, M. Reiher, and L. González, "Orbital entanglement and casscf analysis of the ru-no bond in a ruthenium nitrosyl complex," Phys. Chem. Chem. Phys. 17, 14383-14392 (2015).

${ }^{39}$ K. D. Vogiatzis, D. Ma, J. Olsen, L. Gagliardi, and W. A. De Jong, "Pushing configuration-interaction to the limit: Towards massively parallel mcscf calculations," J. Chem. Phys. 147, 184111 (2017).

${ }^{40}$ V. Krewald and D. A. Pantazis, "Applications of the density matrix renormalization group to exchange-coupled transition metal systems," in Transition Metals in Coordination Environments (Springer, 2019) pp. 91-120.

${ }^{41} \mathrm{~B}$. Huron, J. Malrieu, and P. Rancurel, "Iterative perturbation calculations of ground and excited state energies from multiconfigurational zeroth-order wavefunctions," J. Chem. Phys. 58, 5745-5759 (1973).

${ }^{42}$ J. B. Schriber and F. A. Evangelista, "Communication: An adaptive configuration interaction approach for strongly correlated electrons with tunable accuracy," J. Chem. Phys. 144 (2016).

${ }^{43}$ N. M. Tubman, J. Lee, T. Y. Takeshita, M. Head-Gordon, and K. B. Whaley, "A deterministic alternative to the full configuration interaction quantum monte carlo method," J. Chem. Phys. 145, $044112(2016)$

${ }^{44}$ A. A. Holmes, N. M. Tubman, and C. Umrigar, "Heat-bath configuration interaction: An efficient selected configuration interaction algorithm inspired by heat-bath sampling," J. Chem. Theory Comput. 12, 3674-3680 (2016).

${ }^{45}$ J. J. Eriksen, T. A. Anderson, J. E. Deustua, K. Ghanem, D. Hait, M. R. Hoffmann, S. Lee, D. S. Levine, I. Magoulas, J. Shen, et al., "The ground state electronic energy of benzene," J. Phys. Chem. Lett. 11, 8922-8929 (2020).

${ }^{46}$ P.-F. Loos, Y. Damour, and A. Scemama, "The performance of cipsi on the ground state electronic energy of benzene," J. Chem. Phys. 153, 176101 (2020).

${ }^{47} \mathrm{M}$. A. Ortuño and C. J. Cramer, "Multireference electronic structures of fe-pyridine(diimine) complexes over multiple oxidation states," J. Phys. Chem. A 121, 5932-5939 (2017), http://dx.doi.org/10.1021/acs.jpca.7b06032.

${ }^{48}$ Z. Li, J. Li, N. S. Dattani, C. Umrigar, and G. K.-L. Chan, "The electronic complexity of the ground-state of the femo cofactor of nitrogenase as relevant to quantum simulations," J. Chem. Phys. 150, 024302 (2019).

${ }^{49}$ J. M. Montgomery and D. A. Mazziotti, "Strong electron correlation in nitrogenase cofactor, femoco," J. Phys. Chem. A 122, 4988-4996 (2018).

${ }^{50} \mathrm{~J}$. Lee and M. Head-Gordon, "Regularized orbital-optimized second-order møller-plesset perturbation theory: A reliable fifth-order-scaling electron correlation model with orbital energy dependent regularizers," J. Chem. Theory Comput. 14, 5203-5219 (2018).

${ }^{51}$ C. D. Sherrill, M. S. Lee, and M. Head-Gordon, "On the performance of density functional theory for symmetry-breaking problems," Chem. Phys. Lett. 302, 425-430 (1999).

52 J. Lee and M. Head-Gordon, "Distinguishing artificial and essential symmetry breaking in a single determinant: Approach 
and application to the c 60, c 36, and c 20 fullerenes," Phys. Chem. Chem. Phys. 21, 4763-4778 (2019).

${ }^{53}$ J. Lee and M. Head-Gordon, "Two single-reference approaches to singlet biradicaloid problems: Complex, restricted orbitals and approximate spin-projection combined with regularized orbital-optimized møller-plesset perturbation theory," J. Chem. Phys. 150, 244106 (2019).

${ }^{54}$ J. Lee, F. D. Malone, and M. A. Morales, "Utilizing essential symmetry breaking in auxiliary-field quantum monte carlo: Application to the spin gaps of the c36 fullerene and an iron porphyrin model complex," J. Chem. Theory Comput. 16, 30193027 (2020).

${ }^{55} \mathrm{~L}$. E. Orgel, An introduction to transition-metal chemistry: ligand-field theory (Taylor \& Francis, 1966).

${ }^{56}$ C. C. J. Roothaan, "New developments in molecular orbital theory," Rev. Mod. Phys. 23, 69 (1951).

${ }^{57}$ G. L. Miessler and D. A. Tarr, Inorganic Chemistry, Fourth Edition (Prentice Hall, 2011).

${ }^{58}$ S. K. Singh, J. Eng, M. Atanasov, and F. Neese, "Covalency and chemical bonding in transition metal complexes: An ab initio based ligand field perspective," Coord. Chem. Rev. 344, 2-25 (2017).

${ }^{59}$ L. Lang, M. Atanasov, and F. Neese, "Improvement of ab initio ligand field theory by means of multistate perturbation theory," J. Phys. Chem. A 124, 1025-1037 (2020).

${ }^{60}$ N. M. Tubman, C. D. Freeman, D. S. Levine, D. Hait, M. HeadGordon, and K. B. Whaley, "Modern approaches to exact diagonalization and selected configuration interaction with the adaptive sampling ci method," J. Chem. Theory Comput. 16, 2139-2159 (2020).

${ }^{61}$ N. M. Tubman, D. S. Levine, D. Hait, M. Head-Gordon, and K. B. Whaley, "An efficient deterministic perturbation theory for selected configuration interaction methods," arXiv preprint arXiv:1808.02049 (2018).

${ }^{62}$ S. M. Sharada, D. Stück, E. J. Sundstrom, A. T. Bell, and M. Head-Gordon, "Wavefunction stability analysis without analytical electronic hessians: application to orbital-optimised second-order møller-plesset theory and vv10-containing density functionals," Mol. Phys. 113, 1802-1808 (2015).

${ }^{63} \mathrm{P}$. Mori-Sánchez, A. J. Cohen, and W. Yang, "Manyelectron self-interaction error in approximate density functionals," J. Chem. Phys. 125, 201102 (2006), https://doi.org/10.1063/1.2403848.

${ }^{64}$ J. P. Perdew, R. G. Parr, M. Levy, and J. L. Balduz, "Densityfunctional theory for fractional particle number: Derivative discontinuities of the energy," Phys. Rev. Lett. 49, 1691-1694 (1982).

${ }^{65}$ P.-O. Löwdin, "Quantum theory of many-particle systems. i. physical interpretations by means of density matrices, natural spin-orbitals, and convergence problems in the method of configurational interaction," Phys. Rev. 97, 1474 (1955).

${ }^{66}$ J. Wang, A. D. Becke, and V. H. Smith Jr, "Evaluation of s $_{2}$ i in restricted, unrestricted hartree-fock, and density functional based theories," J. Chem. Phys. 102, 3477-3480 (1995).

${ }^{67}$ A. J. Cohen, D. J. Tozer, and N. C. Handy, "Evaluation of < $s^{2}>$ in density functional theory," J. Chem. Phys. 126, 214104 (2007).

${ }^{68}$ A. I. Krylov, "Spin-contamination of coupled-cluster wave functions," J. Chem. Phys. 113, 6052-6062 (2000).

${ }^{69}$ Y. Shao, Z. Gan, E. Epifanovsky, A. T. Gilbert, M. Wormit, J. Kussmann, A. W. Lange, A. Behn, J. Deng, X. Feng, et al., "Advances in molecular quantum chemistry contained in the q-chem 4 program package," Mol. Phys. 113, 184-215 (2015).

${ }^{70}$ F. Neese, "The orca program system," Wiley Interdiscip. Rev. Comput. Mol. Sci. 2, 73-78 (2012).

${ }^{71}$ D. S. Levine, D. Hait, N. M. Tubman, S. Lehtola, K. B. Whaley, and M. Head-Gordon, "Casscf with extremely large active spaces using the adaptive sampling configuration interaction method," J. Chem. Theory Comput. 16, 2340-2354 (2020).
${ }^{72} \mathrm{~F}$. Weigend and R. Ahlrichs, "Balanced basis sets of split valence, triple zeta valence and quadruple zeta valence quality for h to rn: Design and assessment of accuracy," Phys. Chem. Chem. Phys. 7, 3297-3305 (2005).

${ }^{73} \mathrm{~T}$. Van Voorhis and M. Head-Gordon, "A geometric approach to direct minimization," Mol. Phys. 100, 1713-1721 (2002).

${ }^{74}$ S. Dasgupta and J. M. Herbert, "Standard grids for highprecision integration of modern density functionals: $\mathrm{Sg}-2$ and sg-3," J. Comput. Chem. 38, 869-882 (2017).

${ }^{75}$ R. Z. Khaliullin, E. A. Cobar, R. C. Lochan, A. T. Bell, and M. Head-Gordon, "Unravelling the origin of intermolecular interactions using absolutely localized molecular orbitals," J. Phys. Chem. A 111, 8753-8765 (2007).

${ }^{76}$ P. R. Horn, Y. Mao, and M. Head-Gordon, "Probing noncovalent interactions with a second generation energy decomposition analysis using absolutely localized molecular orbitals," Phys. Chem. Chem. Phys. 18, 23067-23079 (2016).

${ }^{77}$ M. Loipersberger, Y. Mao, and M. Head-Gordon, "Variational forward-backward charge transfer analysis based on absolutely localized molecular orbitals: Energetics and molecular properties," J. Chem. Theory Comput. 16, 1073-1089 (2020).

${ }^{78}$ D. Hait, A. Rettig, and M. Head-Gordon, "Beyond the coulsonfischer point: characterizing single excitation ci and tddft for excited states in single bond dissociations," Phys. Chem. Chem. Phys. 21, 21761-21775 (2019).

${ }^{79}$ C. A. Coulson and I. Fischer, "Xxxiv. notes on the molecular orbital treatment of the hydrogen molecule," Philos. Mag. 40, 386-393 (1949).

${ }^{80}$ L. V. Slipchenko and A. I. Krylov, "Singlet-triplet gaps in diradicals by the spin-flip approach: A benchmark study," J. Chem. Phys. 117, 4694-4708 (2002).

${ }^{81}$ J. Shee, E. J. Arthur, S. Zhang, D. R. Reichman, and R. A. Friesner, "Singlet-triplet energy gaps of organic biradicals and polyacenes with auxiliary-field quantum monte carlo," J. Chem. Theory Comput. 15, 4924-4932 (2019).

${ }^{82}$ Y. A. Bernard, Y. Shao, and A. I. Krylov, "General formulation of spin-flip time-dependent density functional theory using noncollinear kernels: Theory, implementation, and benchmarks," J. Chem. Phys. 136, 204103 (2012).

${ }^{83}$ T. Yanai, D. P. Tew, and N. C. Handy, "A new hybrid exchange-correlation functional using the coulomb-attenuating method (cam-b3lyp)," Chem. Phys. Lett. 393, 51-57 (2004).

${ }^{84}$ L. Kronik, T. Stein, S. Refaely-Abramson, and R. Baer, "Excitation gaps of finite-sized systems from optimally tuned rangeseparated hybrid functionals," J. Chem. Theory Comput. 8, 1515-1531 (2012).

${ }^{85} \mathrm{~J}$. Shee and M. Head-Gordon, "Predicting excitation energies of twisted intramolecular charge-transfer states with the timedependent density functional theory: Comparison with experimental measurements in the gas phase and solvents ranging from hexanes to acetonitrile," J. Chem. Theory Comput. (2020).

${ }^{86} \mathrm{~B}$. Pritchard and J. Autschbach, "Theoretical investigation of paramagnetic nmr shifts in transition metal acetylacetonato complexes: Analysis of signs, magnitudes, and the role of the covalency of ligand-metal bonding," Inorg. Chem. 51, 8340-8351 (2012).

${ }^{87}$ J. Autschbach and M. Srebro, "Delocalization error and "functional tuning" in kohn-sham calculations of molecular properties," Acc. Chem. Res. 47, 2592-2602 (2014).

${ }^{88}$ D. Hait and M. Head-Gordon, "Delocalization errors in density functional theory are essentially quadratic in fractional occupation number," J. Phys. Chem. Lett. 9, 6280-6288 (2018).

${ }^{89}$ C. D. Sherrill, A. I. Krylov, E. F. Byrd, and M. Head-Gordon, "Energies and analytic gradients for a coupled-cluster doubles model using variational brueckner orbitals: application to symmetry breaking in o 4+," J. Chem. Phys. 109, 4171-4181 (1998).

${ }^{90}$ D. Casanova and A. I. Krylov, "Spin-flip methods in quantum chemistry," Phys. Chem. Chem. Phys. 22, 4326-4342 (2020).

${ }^{91} \mathrm{~S}$. C. Rasmussen, "The 18-electron rule and electron counting in transition metal compounds: theory and application," Chem- 
Texts 1, 10 (2015).

${ }^{92}$ A. Domingo, M. Àngels Carvajal, and C. De Graaf, "Spin crossover in fe (ii) complexes: An ab initio study of ligand $\sigma$ donation," Int. J. Quantum Chem. 110, 331-337 (2010).

${ }^{93}$ J. M. Galbraith and H. F. Schaefer III, "Concerning the applicability of density functional methods to atomic and molecular negative ions," J. Chem. Phys. 105, 862-864 (1996).

${ }^{94}$ O. A. Vydrov, G. E. Scuseria, and J. P. Perdew, "Tests of functionals for systems with fractional electron number," J. Chem. Phys. 126, 154109 (2007).

${ }^{95}$ W. R. Scheidt and C. A. Reed, "Spin-state/stereochemical relationships in iron porphyrins: implications for the hemoproteins," Chem. Rev. 81, 543-555 (1981).

${ }^{96} \mathrm{P}$. Gütlich and H. A. Goodwin, "Spin crossover - an overall perspective," in Spin Crossover in Transition Metal Compounds I (Springer, 2004) pp. 1-47.

97 J.-F. Létard, P. Guionneau, and L. Goux-Capes, "Towards spin crossover applications," in Spin Crossover in Transition Metal Compounds III (Springer, 2004) pp. 221-249.

${ }^{98}$ D. A. Reed, B. K. Keitz, J. Oktawiec, J. A. Mason, T. Runčevski, D. J. Xiao, L. E. Darago, V. Crocellà, S. Bordiga, and J. R. Long, "A spin transition mechanism for cooperative adsorption in metal-organic frameworks," Nature 550, 96-100 (2017).

${ }^{99}$ L. M. Lawson Daku, F. Aquilante, T. W. Robinson, and A. Hauser, "Accurate spin-state energetics of transition metal complexes. 1. ccsd (t), caspt2, and dft study of [m (nch) 6] $2+(\mathrm{m}=$ fe, co)," J. Chem. Theory Comput. 8, 4216-4231 (2012).

${ }^{100}$ S. Song, M.-C. Kim, E. Sim, A. Benali, O. Heinonen, and K. Burke, "Benchmarks and reliable dft results for spin gaps of small ligand fe (ii) complexes," J. Chem. Theory Comput. 14, 2304-2311 (2018).

${ }^{101}$ A. Droghetti, D. Alfè, and S. Sanvito, "Assessment of density functional theory for iron (ii) molecules across the spin-crossover transition," J. Chem. Phys. 137, 124303 (2012).

${ }^{102}$ B. M. Flöser, Y. Guo, C. Riplinger, F. Tuczek, and F. Neese, "Detailed pair natural orbital-based coupled cluster studies of spin crossover energetics," J. Chem. Theory Comput. 16, 22242235 (2020).

${ }^{103}$ K. Yamaguchi, F. Jensen, A. Dorigo, and K. Houk, "A spin correction procedure for unrestricted hartree-fock and møllerplesset wavefunctions for singlet diradicals and polyradicals," Chem. Phys. Lett. 149, 537-542 (1988).

${ }^{104}$ S. Yamanaka, T. Kawakami, H. Nagao, and K. Yamaguchi, "Effective exchange integrals for open-shell species by density functional methods," Chem. Phys. Lett. 231, 25-33 (1994).

${ }^{105}$ T. F. Hughes and R. A. Friesner, "Development of accurate dft methods for computing redox potentials of transition metal complexes: results for model complexes and application to cytochrome p450," J. Chem. Theory Comput. 8, 442-459 (2012).

${ }^{106}$ L. E. Roy, E. Jakubikova, M. G. Guthrie, and E. R. Batista, "Calculation of one-electron redox potentials revisited. is it possible to calculate accurate potentials with density functional methods?" J. Phys. Chem. A 113, 6745-6750 (2009).

${ }^{107}$ M. Beley, J.-P. Collin, R. Ruppert, and J.-P. Sauvage, "Nickel (ii)-cyclam: an extremely selective electrocatalyst for reduction of co 2 in water," Chem. Comm. , 1315-1316 (1984).

${ }^{108}$ T. Geiger and F. C. Anson, "Homogeneous catalysis of the electrochemical reduction of dioxygen by a macrocyclic cobalt (iii) complex," J. Am. Chem. Soc. 103, 7489-7496 (1981).

${ }^{109}$ E. Tsuchida, K. Oyaizu, E. L. Dewi, T. Imai, and F. C. Anson, "Catalysis of the electroreduction of $\mathrm{o} 2$ to h2o by vanadiumsalen complexes in acidified dichloromethane," Inorg. Chem. 38, 3704-3708 (1999).

${ }^{110}$ Z. Liu and F. C. Anson, "Electrochemical properties of vanadium (iii, iv, v)- salen complexes in acetonitrile. four-electron reduction of o2 by v (iii)- salen," Inorg. Chem. 39, 274-280 (2000).

${ }^{111}$ J. Song, E. L. Klein, F. Neese, and S. Ye, "The mechanism of homogeneous co 2 reduction by ni(cyclam): Product selectiv- ity, concerted proton-electron transfer and c-o bond cleavage," Inorg. Chem. 53, 7500-7507 (2014).

${ }^{112}$ I. Bhugun, D. Lexa, and J.-M. Savéant, "Catalysis of the electrochemical reduction of carbon dioxide by iron (0) porphyrins: Synergystic effect of weak brönsted acids," J. Am. Chem. Soc. 118, 1769-1776 (1996).

${ }^{113}$ J. P. Collman, S. Ghosh, A. Dey, R. A. Decréau, and Y. Yang, "Catalytic reduction of o2 by cytochrome c using a synthetic model of cytochrome c oxidase," J. Am. Chem. Soc. 131, 50345035 (2009).

${ }^{114}$ Z. Halime, H. Kotani, Y. Li, S. Fukuzumi, and K. D. Karlin, "Homogeneous catalytic o2 reduction to water by a cytochrome c oxidase model with trapping of intermediates and mechanistic insights," Proc. Natl. Acad. Sci. 108, 13990-13994 (2011).

${ }^{115}$ C. Costentin, S. Drouet, M. Robert, and J.-M. Savéant, "A local proton source enhances co2 electroreduction to co by a molecular fe catalyst," Science 338, 90-94 (2012).

${ }^{116}$ C. Römelt, J. Song, M. Tarrago, J. A. Rees, M. van Gastel, T. Weyhermüller, S. DeBeer, E. Bill, F. Neese, and S. Ye, "Electronic structure of a formal iron (0) porphyrin complex relevant to co2 reduction," Inorg. Chem. 56, 4745-4750 (2017).

${ }^{117}$ E. E. Benson, M. D. Sampson, K. A. Grice, J. M. Smieja, J. D. Froehlich, D. Friebel, J. A. Keith, E. A. Carter, A. Nilsson, and C. P. Kubiak, "The electronic states of rhenium bipyridyl electrocatalysts for co2 reduction as revealed by x-ray absorption spectroscopy and computational quantum chemistry," Angew. Chem. Int. Ed. 52, 4841-4844 (2013).

${ }^{118}$ C. Riplinger, M. D. Sampson, A. M. Ritzmann, C. P. Kubiak, and E. A. Carter, "Mechanistic contrasts between manganese and rhenium bipyridine electrocatalysts for the reduction of carbon dioxide," J. Am. Chem. Soc. 136, 16285-16298 (2014).

${ }^{119}$ M. Loipersberger, D. Z. Zee, J. A. Panetier, C. J. Chang, J. R. Long, and M. Head-Gordon, "Computational study of an iron(ii) polypyridine electrocatalyst for co2 reduction: Key roles for intramolecular interactions in co2 binding and proton transfer," Inorg. Chem. 59, 8146-8160 (2020).

${ }^{120}$ J. S. Derrick, M. Loipersberger, R. Chatterjee, D. A. Iovan, P. T. Smith, K. Chakarawet, J. Yano, J. R. Long, M. Head-Gordon, and C. J. Chang, "Metal-ligand cooperativity via exchange coupling promotes iron- catalyzed electrochemical co2 reduction at low overpotentials," J. Am. Chem. Soc. 142, 20489-20501 (2020).

${ }^{121}$ M. Loipersberger, D. G. A. Cabral, D. B. K. Chu, and M. HeadGordon, "Mechanistic insights into co and fe quaterpyridinebased co2 reduction catalysts: Metal-ligand orbital interaction as the key driving force for distinct pathways," J. Am. Chem. Soc. 143, 744-763 (2021).

${ }^{122}$ B. Butschke, K. L. Fillman, T. Bendikov, L. J. W. Shimon, Y. Diskin-Posner, G. Leitus, S. I. Gorelsky, M. L. Neidig, and D. Milstein, "How innocent are potentially redox non-innocent ligands? electronic structure and metal oxidation states in iron-pnn complexes as a representative case study," Inorg. Chem. 54, 4909-4926 (2015), http://dx.doi.org/10.1021/acs.inorgchem.5b00509.

${ }^{123} \mathrm{R}$. H. Crabtree, The organometallic chemistry of the transition metals (John Wiley \& Sons, 2009).

${ }^{124}$ S. Sreekantan Nair Lalithambika, R. Golnak, B. Winter, and K. Atak, "Electronic structure of aqueous [co (bpy) 3] 2+/3+ electron mediators," Inorg. Chem. 58, 4731-4740 (2019).

${ }^{125}$ J. A. Keith, K. A. Grice, C. P. Kubiak, and E. A. Carter, "Elucidation of the selectivity of proton-dependent electrocatalytic co2 reduction by fac-re (bpy)(co) 3cl," J. Am. Chem. Soc. 135, 15823-15829 (2013).

${ }^{126}$ D. Z. Zee, M. Nippe, A. E. King, C. J. Chang, and J. R. Long, "Tuning second coordination sphere interactions in polypyridyliron complexes to achieve selective electrocatalytic reduction of carbon dioxide to carbon monoxide," Inorg. Chem. 59, 52065217 (2020).

${ }^{127}$ I. Azcarate, C. Costentin, M. Robert, and J.-M. Savéant, "Through-space charge interaction substituent effects in molecu- 
lar catalysis leading to the design of the most efficient catalyst of co2-to-co electrochemical conversion," J. Am. Chem. Soc. 138, 16639-16644 (2016).

${ }^{128}$ J. P. Collman, J. Hoard, N. Kim, G. Lang, and C. A. Reed, "Synthesis, stereochemistry, and structure-related properties of. alpha.,. beta.,. gamma.,. delta.-tetraphenylporphinatoiron (ii)," J. Am. Chem. Soc. 97, 2676-2681 (1975)

${ }^{129}$ H. Chen, W. Lai, and S. Shaik, "Multireference and multiconfiguration ab initio methods in heme-related systems: What have we learned so far?" J. Phys. Chem. B 115, 1727-1742 (2011).

${ }^{130} \mathrm{M}$. Radon and K. Pierloot, "Binding of co, no, and o2 to heme by density functional and multireference ab initio calculations," J. Phys. Chem. A 112, 11824-11832 (2008).

${ }^{131}$ M. Radon, E. Broclawik, and K. Pierloot, "Electronic structure of selected $\{\mathrm{FeNO}\} 7$ complexes in heme and non-heme architectures: A density functional and multireference ab initio study," J. Phys. Chem. B 114, 1518-1528 (2010).

${ }^{132}$ K. Boguslawski, C. R. Jacob, and M. Reiher, "Can dft accurately predict spin densities? analysis of discrepancies in iron nitrosyl complexes," J. Chem. Theory Comput. 7, 2740-2752 (2011).

${ }^{133}$ P. J. Hay, J. C. Thibeault, and R. Hoffmann, "Orbital interactions in metal dimer complexes," J. Am. Chem. Soc. 97, 48844899 (1975).

${ }^{134} \mathrm{O}$. Kahn and B. Briat, "Exchange interaction in polynuclear complexes. part 1.- principles, model and application to the binuclear complexes of chromium (iii)," J. Chem. Soc., Faraday Trans. 2 72, 268-281 (1976).

${ }^{135} \mathrm{O}$. Kahn and B. Briat, "Exchange interaction in polynuclear complexes. part 2.-antiferromagnetic coupling in binuclear oxo-bridged iron (iii) complexes," J. Chem. Soc., Faraday Trans. 2 72, 1441-1446 (1976).

${ }^{136}$ M. Orio, D. A. Pantazis, T. Petrenko, and F. Neese, "Magnetic and spectroscopic properties of mixed valence manganese (iii, iv) dimers: a systematic study using broken symmetry density functional theory," Inorg. Chem. 48, 7251-7260 (2009).

${ }^{137}$ D. A. Pantazis, "Assessment of double-hybrid density functional theory for magnetic exchange coupling in manganese complexes," Inorganics 7, 57 (2019).

${ }^{138}$ K. E. Lewis, D. M. Golden, and G. P. Smith, "Organometallic bond dissociation energies: laser pyrolysis of iron pentacarbonyl, chromium hexacarbonyl, molybdenum hexacarbonyl, and tungsten hexacarbonyl," J. Am. Chem. Soc. 106, 3905-3912 (1984).

139 J. P. Perdew, A. Ruzsinszky, J. Tao, V. N. Staroverov, G. E. Scuseria, and G. I. Csonka, "Prescription for the design and selection of density functional approximations: More constraint satisfaction with fewer fits," J. Chem. Phys. 123, 062201 (2005).

${ }^{140}$ N. Mardirossian and M. Head-Gordon, "Thirty years of density functional theory in computational chemistry: an overview and extensive assessment of 200 density functionals," Mol. Phys. 115, 2315-2372 (2017).
${ }^{141}$ C. van Wüllen, "A relativistic kohn-sham density functional procedure by means of direct perturbation theory. ii. application to the molecular structure and bond dissociation energies of transition metal carbonyls and related complexes," J. Chem. Phys. 105, 5485-5493 (1996).

${ }^{142}$ F. Neese, T. Schwabe, S. Kossmann, B. Schirmer, and S. Grimme, "Assessment of orbital-optimized, spin-component scaled second-order many-body perturbation theory for thermochemistry and kinetics," J. Chem. Theory Comput. 5, 30603073 (2009).

${ }^{143}$ I. Hyla-Kryspin and S. Grimme, "Comprehensive study of the thermochemistry of first-row transition metal compounds by spin component scaled mp2 and mp3 methods," Organometallics 23, 5581-5592 (2004).

${ }^{144}$ T. Stuyver, B. Chen, T. Zeng, P. Geerlings, F. De Proft, and R. Hoffmann, "Do diradicals behave like radicals?" Chem. Rev. 119, 11291-11351 (2019).

${ }^{145}$ G. J. Beran, S. R. Gwaltney, and M. Head-Gordon, "Approaching closed-shell accuracy for radicals using coupled cluster theory with perturbative triple substitutions," Phys. Chem. Chem. Phys. 5, 2488-2493 (2003).

${ }^{146}$ L. W. Bertels, J. Lee, and M. Head-Gordon, "Polishing the gold standard: The role of orbital choice in ccsd ( $\mathrm{t}$ ) vibrational frequency prediction," J. Chem. Theory Comput. (2020).

${ }^{147}$ G. P. Chen, V. K. Voora, M. M. Agee, S. G. Balasubramani, and F. Furche, "Random-phase approximation methods," Annu. Rev. Phys. Chem. 68, 421-445 (2017).

${ }^{148}$ H. Eshuis, J. Yarkony, and F. Furche, "Fast computation of molecular random phase approximation correlation energies using resolution of the identity and imaginary frequency integration," J. Chem. Phys. 132, 234114 (2010).

${ }^{149}$ G. E. Scuseria, T. M. Henderson, and D. C. Sorensen, "The ground state correlation energy of the random phase approximation from a ring coupled cluster doubles approach," (2008).

${ }^{150} \mathrm{~J}$. Bates, P. Mezei, G. Csonka, J. Sun, and A. Ruzsinszky, "Reference determinant dependence of the random phase approximation in $3 \mathrm{~d}$ transition metal chemistry," J. Chem. Theory Comput. 13, 100-109 (2017).

${ }^{151}$ P. Mori-Sánchez, A. J. Cohen, and W. Yang, "Failure of the random-phase-approximation correlation energy," Phys. Rev. A 85, 042507 (2012).

${ }^{152}$ Y. Jin, D. Zhang, Z. Chen, N. Q. Su, and W. Yang, "Generalized optimized effective potential for orbital functionals and self-consistent calculation of random phase approximations," J. Phys. Chem. Lett. 8, 4746-4751 (2017).

${ }^{153}$ S. Grimme and M. Steinmetz, "A computationally efficient double hybrid density functional based on the random phase approximation," Phys. Chem. Chem. Phys. 18, 20926-20937 (2016). 